\title{
Higher-order glass-transition singularities in colloidal systems with attractive interactions
}

\author{
K. Dawson, ${ }^{1}$ G. Foffi, ${ }^{1}$ M. Fuchs, ${ }^{2}$ W. Götze,${ }^{2}$ F. Sciortino, ${ }^{3}$ M. Sperl, ${ }^{2}$ P. Tartaglia, ${ }^{3}$ Th. Voigtmann, ${ }^{2}$ and E. Zaccarelli ${ }^{1}$ \\ ${ }^{1}$ Irish Centre for Colloid Science and Biomaterials, University College Dublin, Belfield, Dublin 4, Ireland \\ ${ }^{2}$ Physik-Department, Technische Universität München, D-85747 Garching, Germany \\ ${ }^{3}$ Dipartimento di Fisica and Istituto Nazionale per la Fisica della Materia, Università di Roma La Sapienza, \\ Piazzale Aldo Moro 2, I-00185 Roma, Italy
}

(Received 20 June 2000; published 19 December 2000)

\begin{abstract}
The transition from a liquid to a glass in colloidal suspensions of particles interacting through a hard core plus an attractive square-well potential is studied within the mode-coupling-theory framework. When the width of the attractive potential is much shorter than the hard-core diameter, a reentrant behavior of the liquid-glass line and a glass-glass-transition line are found in the temperature-density plane of the model. For small well-width values, the glass-glass-transition line terminates in a third-order bifurcation point, i.e., in a $A_{3}$ (cusp) singularity. On increasing the square-well width, the glass-glass line disappears, giving rise to a fourthorder $A_{4}$ (swallow-tail) singularity at a critical well width. Close to the $A_{3}$ and $A_{4}$ singularities the decay of the density correlators shows stretching of huge dynamical windows, in particular logarithmic time dependence.
\end{abstract}

PACS number(s): 82.70.Dd, 61.20.Ne, 64.70.Pf

\section{INTRODUCTION}

Colloidal suspensions have been studied extensively because of their practical importance and because of their relevance in biophysics. These systems are also of great theoretical interest since they are models for conventional matter. They can be prepared for a large span of densities so that the states can be gases, gels, liquids, crystalline solids, or glasses. Light scattering can be used to measure the static structure factor and various correlation functions. The dynamics can be explored over a wide range of length scales and over huge dynamical windows $[1,2]$. It is fascinating that with colloidal systems the interaction can be tuned to some extent by varying the coating of the particles and the composition of the solvent [1-3]. It is possible to realize the hard-sphere system (HSS), the basic model underlying all theories of simple liquids [4,5]. One can also prepare systems where the hard core is complemented by an attractive shell. This allows one to study the interplay of repulsion and attraction. As a contribution to such studies, a theory for the glass formation resulting from a strong short-range attraction among densely packed hard-sphere colloidal particles shall be presented in this paper.

In hard-sphere colloidal dispersions, the liquid-glass transition has been studied by van Megen and Pusey [6]. They measured correlation functions $\phi_{q}(t)$ for density fluctuations of a representative set of wave numbers $q$ over about four decades in time $t$. It was found that these correlations decay to zero as expected for a liquid only for packing fractions $\varphi$ below a critical value $\varphi_{c}$. At $\varphi_{c}$, the long-time limit of the correlators, $f_{q}=\phi_{q}(t \rightarrow \infty)$, changes discontinuously to a certain value $f_{q}^{c}>0$, increasing further with the packing fraction. $f_{q}$ is the Debye-Waller factor of the amorphous solid, i.e., of the glass, and generalizes the order parameter introduced by Edwards and Anderson in the theory of spin glasses [7]. The evolution of the glassy dynamics for the HSS was studied comprehensively by van Megen and coworkers [8-13]. The data suggest that it is the well-known

Konstanzer Online-Publikations-System (KOPS)

URL: http://www.ub.uni-konstanz.de/kops/volltexte/2007/3678/

URN: http://nbn-resolving.de/urn:nbn:de:bsz:352-opus-36788 cage effect [5] which causes the glassy dynamics and the arrest of density fluctuations at $\varphi_{c}$.

The cage effect is the essential physical concept underlying the mode-coupling theory (MCT) for the evolution of glassy dynamics in simple systems $[14,15]$. This theory allows the calculation of $\phi_{q}(t)$ and thus $f_{q}$ from the equilibrium structure factor $S_{q}$. As a function of control parameters like $\varphi$, singularities of $f_{q}$, called glass-transition singularities, may occur. The simplest type, called a fold bifurcation, describes a liquid-glass transition at $\varphi=\varphi_{c}$. It implies a subtle dynamical scenario, giving rise to universal features of glassy dynamics which have been identified in a leadingorder-asymptotic expansion of the MCT equations. A review of the basic results is given in Ref. [16]. In Refs. [8-12] detailed quantitative comparisons of the data for hard-sphere colloids with the MCT predictions are presented. It is shown that the theory accounts for the experimental facts within a $15 \%$-accuracy level. An illuminating summary of these studies is given in Ref. [17]. Results for the shear modulus have also been interpreted with the universal MCT formulas [18]. The evolution of glassy dynamics for $\varphi$ increasing towards $\varphi_{c}$ was also studied for polymer micronetwork colloids [1922]. Here, the interparticle interaction is not known. But the authors demonstrated that a consistent fit of their data with the universal MCT formulas was possible. Preliminary studies of the glassy dynamics of charge-stabilized colloids indicate that these data can also be explained within the MCT [23]. The reported findings shall be taken as a justification to base the theory in this paper on the MCT for simple systems.

Our studies deal with the square-well system (SWS), characterized by a hard-core repulsion for interparticle distances $r<d$, and by a constant attraction potential within the shell $d<r<d+\Delta$. The theory focuses on the high-density regime, say $\varphi>0.4$, so that the cage effect is essential for the dynamics. The relative attraction-shell width $\delta=\Delta / d$ is assumed to be small, say $\delta<0.15$. The main outcome of our theory is the prediction of a higher-order glass-transition singularity at a critical packing fraction $\varphi^{*}$ somewhat above the 
critical point $\varphi_{c}$ of the hard-sphere system and a critical width $\delta^{*}$ of about 0.04 . This singularity organizes a subtle phase diagram and opens up various possibilities for glassy relaxation. The results reflect the interplay of two mechanisms for particle localization, i.e., for the arrest of density fluctuations. It can either be dominated by repulsion of the particle by its cage-forming neighbors, or by the formation of bonds to the boundaries of the cage. Preliminary calculations $[24,25]$ based upon Baxter's adhesive hard-sphere model [27] hinted at some findings to be derived in this paper. Baxter's model treats the limit $\delta \rightarrow 0$, so it cannot deal with the indicated singularity at $\delta^{*}$. Moreover, taken literally, the Baxter model cannot be used as a basis for MCT applications since there appears a divergency due to excitations with large wave vectors. The results for this model in Refs. [24-26] depend in an ill-defined manner on the large$q$ cutoff used there, a problem which is avoided with the SWS.

Dense systems of colloidal particles characterized by a hard core and strong attractions of a range smaller than the core diameter by a factor of at least 10 were realized experimentally, when adding nonadsorbing polymers to either a suspension of colloidal hard spheres [28] or to emulsions [30] in solutions of sterically stabilized particles when decreasing the solvent quality [31-34], and in copolymer micellar systems when changing the temperature [35]. Such systems were also studied in Monte Carlo simulations $[36,37]$. Nonequilibrium phenomena characterized by a number of aspects were found which cannot be understood from the glassy states formed in hard-sphere solutions. First, amorphous solids could be formed by increasing the attraction strength even though the packing fraction was kept fixed well below the value of the hard-sphere glass transition [3034]. Second, due to increasing the strength of a short-ranged attraction by adding small polymers, melting of the glass states was reported for the colloid-polymer mixtures $[29,28]$. Third, the nondecaying frozen structures that were seen when immersing polymer-coated colloidal particles into solvents of decreasing quality [32] exhibited a much larger Debye-Waller factor at small wave vectors than hard-sphere systems. This indicates a much higher rigidity of the solid states on intermediate length scales. In support of this observation, viscoelastic measurements for intermediate frequencies found strongly concentration-dependent elastic moduli $[30,31,33,34]$. It will be shown that our results provide a qualitative explanation of the reported findings.

The paper is organized as follows. In Sec. II we report our results for the structure factor of the SWS and discuss those features that cause various qualitative results of the MCT solutions. Section III presents the main result of this paper, showing the phase diagram and discussing the properties of the glass states resulting from the interplay between attraction and repulsion. In Sec. IV we present some results for the dynamics which illustrate that the higher-order glasstransition singularities cause relaxation stretching which is much more pronounced than is known for the HSS. Section $\mathrm{V}$ presents some concluding remarks.

\section{STRUCTURE FACTOR CALCULATIONS}

\section{A. The model}

The structure factor $S_{q}$ is the essential input information needed to formulate the MCT equations. In this section, $S_{q}$ shall be discussed for the square-well system (SWS). Only such states shall be considered for which $S_{q}$ depends smoothly on the particle density $\rho$, on the temperature $T$, and on the wave number $q$. The interaction potential $V(r)$ for particles with separation distance $r$ consists of a hard-core repulsion for $r<d$, and it has the negative value $-u_{0}$ within the attraction shell $d<r<d+\Delta$. The structure can be specified by three control parameters: the packing fraction $\varphi$ of the hard cores, the ratio $\theta$ of thermal and attractive energy, and the relative width $\delta$ of the attraction shell,

$$
\varphi=\pi \rho d^{3} / 6, \quad \theta=k_{B} T / u_{0}, \quad \delta=\Delta / d .
$$

Let us note the standard concepts needed for a discussion of $S_{q}$ [4]. $g(r)$ and $h(r)=g(r)-1$ abbreviate the pair distribution and the total correlation function, respectively. The Fourier transform $h_{q}$ of the latter determines the structure factor $S_{q}=1+\rho h_{q}$. The Ornstein-Zernike equation formulates an integral equation for $h(r)$, where the kernel is the direct correlation function $c(r)$. In the wave vector domain, it reads $S_{q}=1 /\left[1-\rho c_{q}\right]$, where

$$
c_{q}=\frac{4 \pi}{q} \int_{0}^{\infty} d r \sin (q r)[r c(r)] .
$$

Baxter's method of the Wiener-Hopf factorization $[4,38]$ shall be used to reformulate the Ornstein-Zernike equation. The basic concept of this theory is the factor function $Q(r)$. It is defined as a continuous real function for $r \geqslant 0$, determining $S_{q}$ via its Fourier transform:

$$
\begin{gathered}
S_{q}^{-1}=\hat{Q}(q) \hat{Q}(q)^{*}, \\
\hat{Q}(q)=1-2 \pi \rho \int_{0}^{\infty} d r \exp (i q r) Q(r) .
\end{gathered}
$$

It is anticipated that $Q(r)$ as well as $c(r)$ vanishes beyond a certain distance $R$. For $0 \leqslant r \leqslant R$, there holds

$$
r c(r)=-Q^{\prime}(r)+2 \pi \rho \int_{r}^{R} d s Q^{\prime}(s) Q(s-r) .
$$

Furthermore, one finds for $r>0$

$$
r h(r)=-Q^{\prime}(r)+2 \pi \rho \int_{0}^{R} d s(r-s) h(|r-s|) Q(s) .
$$

For the SWS, $g(r)=0$ is fulfilled for $0<r<d$, and therefore, using $h(r)=g(r)-1$, Eq. (5) splits into three subequations. Most simple is the result for the middle part, $\Delta \leqslant r$ $\leqslant d$, where the formula known from the theory for the hardsphere system (HSS) is reproduced,

$$
Q^{\prime}(r)=a r+b .
$$


Here, coefficients $a$ and $b$ are introduced by

$$
a=1-2 \pi \rho \int_{0}^{d+\Delta} d s Q(s), \quad b=2 \pi \rho \int_{0}^{d+\Delta} d s s Q(s) .
$$

Writing $G(r)=r g(r)$, one finds for small distances, $0 \leqslant r$ $\leqslant \Delta$,

$$
Q^{\prime}(r)=a r+b-2 \pi \rho \int_{r+d}^{d+\Delta} d s G(s-r) Q(s)
$$

and for the attraction shell, $d \leqslant r \leqslant d+\Delta$, one obtains

$$
Q^{\prime}(r)=a r+b-G(r)+2 \pi \rho \int_{0}^{r-d} d s G(r-s) Q(s) .
$$

Some approximation for $c(r)$ has to be introduced into Eq. (4) in order to close the system of Eqs. (4) and (6). In this paper the Percus-Yevick approximation (PYA) and the mean-spherical approximation (MSA) shall be applied [4]. Nezbeda already studied the structure factor for the SWS using the PYA for small well widths $[39,40]$. His equations could be solved only in a restricted region of parameters. Since the boundary of this region of applicability is close to the parameter region $\varphi \approx 0.5, \theta \approx 1$ of interest in this paper, it does not seem appropriate to base the following calculations on these results.

\section{B. Approximations}

Within the PYA, one writes $c(r)=g(r)\{1$ $\left.-\exp \left[V(r) / k_{B} T\right]\right\}$ outside the hard core. Substitution of this ansatz into Eq. (4) and using Eq. (6d) leads to the approximation for $d \leqslant r \leqslant d+\Delta$,

$$
\begin{aligned}
e^{-u_{0} / k_{B} T} G(r)= & a r+b-2 \pi \rho \int_{r}^{d+\Delta} d s Q^{\prime}(s) Q(s-r) \\
& +2 \pi \rho \int_{0}^{r-d} d s G(r-s) Q(s) .
\end{aligned}
$$

Equations (6) and (7) for $Q(r)$ and $G(r)$ are solved numerically. To proceed, the equations are discretized straightforwardly. On each of the three $r$ intervals, a grid of equally spaced points $r_{n}$ is chosen, where $n=1,2, \ldots, 1000$. The functions $Q^{\prime}(r)$ and $G(r)$ are calculated iterating Eqs. (6) and (7). At each step, the function $Q(r)$ is evaluated from $Q^{\prime}(r)$ using a five-point numerical integration. The procedure is carried out until the difference between two successive iterations summed over all points of the $r$ grid becomes less than $10^{-12}$. The integral in Eq. (3a) is determined by a simplified Filon procedure to obtain $\hat{Q}(q)$ and hence $S_{q}$.

The MSA uses $c(r)=-V(r) / k_{B} T$ outside the hard core. Substituting this ansatz into Eq. (4), after integration one obtains for $d \leqslant r \leqslant d+\Delta$

$$
Q(r)=2 \pi \rho \int_{r}^{d+\Delta} d s Q(s) Q(s-r)+\left[(d+\Delta)^{2}-r^{2}\right] /(2 \theta) .
$$

Equations (6) and (8) are solved analytically in a leadingand next-to-leading-order expansion, using the well width $\delta$ as the small parameter. For the organization of the expansion, the quantity $K=\delta / \theta$ is considered fixed. This procedure is motivated by Baxter's theory of sticky hard spheres [27]. He evaluated $S_{q}$ in the limit $\delta \rightarrow 0, u_{0} \rightarrow \infty$, keeping a parameter equivalent to $K$ fixed. Details of the calculation can be found in the Appendix. The hard-core diameter $d$ shall be used as the unit of length. For $\delta \leqslant r \leqslant 1$, the factor function is the parabola known from the theory of the HSS,

$$
Q(r)=a r^{2} / 2+b r+c,
$$

with $a, b$, and $c$ now being smooth functions of the SWS control parameters. For $0 \leqslant r \leqslant \delta$, there is an enhancement above this parabola,

$$
Q(r)=a r^{2} / 2+b r+c+2 \varphi K^{2} \delta[1-(r / \delta)]^{3} .
$$

Within the attraction shell, the leading-order result describes a linear decrease of $Q(r)$ from $K$ to zero. The leading correction adds a quadratic modification. One finds for $1 \leqslant r$ $\leqslant 1+\delta$

$$
\begin{aligned}
Q(r)= & K\left[1-\frac{r-1}{\delta}\right]+K \delta\left\{\frac{1}{2}\left[1-\left(\frac{r-1}{\delta}\right)^{2}\right]\right. \\
& \left.+6 \varphi c_{0}\left[1-\frac{r-1}{\delta}\right]^{2}\right\} .
\end{aligned}
$$

Here and in the following we denote the constants from Eqs. (9) as $a=a_{0}+K \delta a_{1}, b=b_{0}+K \delta b_{1}$, and $c=c_{0}+K \delta c_{1}$. The leading-order contributions are the result of the Baxter limit $\delta \rightarrow 0$,

$$
\begin{gathered}
a_{0}=\left[\frac{1+2 \varphi}{(1-\varphi)^{2}}\right]-\frac{12 K \varphi}{(1-\varphi)}, \\
b_{0}=\left[\frac{-3 \varphi}{2(1-\varphi)^{2}}\right]+\frac{6 K \varphi}{(1-\varphi)}, \\
c_{0}=\left[\frac{-1}{2(1-\varphi)}\right]+K .
\end{gathered}
$$

The terms in large square brackets exhibit the results for the HSS [4]. The coefficients of the next-to-leading-order contributions are 


$$
\begin{gathered}
a_{1}=\left[6 \varphi(5 \varphi-2)-72 c_{0} \varphi^{2}(1-\varphi)\right] /(1-\varphi)^{2}, \\
b_{1}=\left[9 \varphi(1-2 \varphi)+36 c_{0} \varphi^{2}(1-\varphi)\right] /(1-\varphi)^{2}, \\
c_{1}=\left[1-7 \varphi+12 c_{0} \varphi(1-\varphi)\right] /[2(1-\varphi)] .
\end{gathered}
$$

Substitution of Eqs. (9) into Eq. (3a) yields $\hat{Q}(q)$ as a combination of trigonometric functions. It is elementary to work out the somewhat lengthy expression and thus via Eq. (3a) the desired result for $S_{q}$.

The large- $q$ asymptote of the direct correlation function $c_{q}$ shall be obtained from Eq. (2) by evaluating the asymptote of the Fourier-sine transform of the function $f(r)$ $=r c(r)$. From Eq. (4) one concludes that $f(r)$ is smooth except for at most three points $r^{(1)}=\delta, r^{(2)}=1$, and $r^{(3)}=1$ $+\delta$. At these points there can be a discontinuity, given by that of the derivative of the factor function: $f^{(i)}=Q^{\prime}\left(r^{(i)}\right.$ $-0)-Q^{\prime}\left(r^{(i)}+0\right)$. Let us also note from Eq. (4) the initial value $f(r=0)=A=-Q^{\prime}(0)-6 \varphi Q(0)^{2}$. For the exact solution of the problem, $A=0$ must hold, but due to the approximation scheme used here, a finite value of $\mathcal{O}(\delta)$ remains: $A=-K \delta\left[12 \varphi c_{0}\left(c_{1}+2 K \varphi\right)+b_{1}\right]+\mathcal{O}\left(\delta^{2}\right)$. Thereby, the Baxter result [27], $A=\mathcal{O}\left(\delta^{0}\right)$, is improved. The $f^{(i)}$ can be determined easily from Eqs. (9), in particular $f^{(1)}=0$. Integrating by parts, the integral in Eq. (2) becomes $\left[f^{(0)}\right.$ $\left.+\Sigma_{i} f^{(i)} \cos \left(q r^{(i)}\right)\right] / q+\mathcal{O}\left(1 / q^{2}\right)$. Hence one arrives at $c_{q}$ $=c_{q}^{\text {as }}+\mathcal{O}\left(1 / q^{3}\right)$, where the asymptotic tail reads

$c_{q}^{\mathrm{as}}=\left(4 \pi / q^{2}\right)\{A+B \cos (q)+2 C \sin [q(1+\delta / 2)] \sin (q \delta / 2)\}$.

The second term in the curly brackets has a form familiar from the PYA result for the HSS. But the coefficient $B$ is a smooth function of $K$ and $\delta$ which reduces to the HSS value for $K=\delta=0$,

$$
B=a+b+K\left(12 \varphi c_{0}-1\right) .
$$

The third term in the curly bracket is due to the existence of the attraction well. Its prefactor reads

$$
C=(1+\delta) / \theta
$$

\section{Results}

The spinodal lines of the SWS are shown in Fig. 1 for three representative values of the well width $\delta$. They specify the divergence points of the compressibility, i.e., the zeros of $S_{q}^{-1}$ for $q=0$. The spinodal is the boundary of the regime of absolute instability with respect to the liquid-vapor transition. Only states outside this regime can be considered in the following. Substitution of Eqs. (9) into Eq. (3b) yields elementary expressions for $\hat{Q}(0)$ within the MSA. We have not been able to determine the spinodals within the PYA due to numerical instability of the algorithm. The high-density regime investigated in the following applications of the MCT is indicated as the strip between the two dotted vertical lines.

Figure 2 exhibits the structure factors $S_{q}$, calculated within the MSA for $\delta=0.05$, and the corresponding pair distributions $g(r)$, calculated numerically from Eq. (5), for

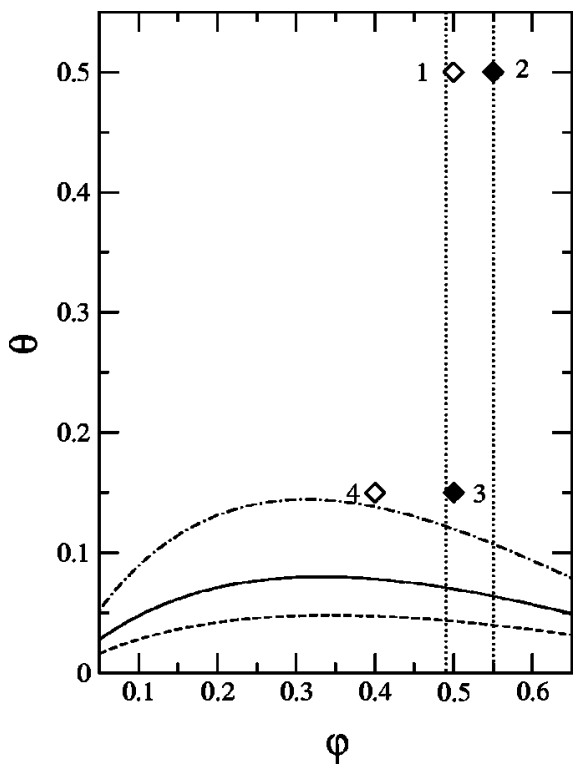

FIG. 1. Control-parameter plane for the square-well system (SWS) plotted as dimensionless temperature $\theta=k_{B} T / u_{0}$ versus packing fraction $\varphi$. The full line shows the spinodal calculated within the MSA for the relative attraction-well width $\delta=0.05$. Dashed (dash-dotted) curves show the corresponding spinodals for $\delta=0.03(\delta=0.09)$. Vertical dotted lines mark the region for which the phase diagram is discussed below in Fig. 5. Diamonds mark the state parameters for which the structure factor is shown in Fig. 2.

states marked by diamonds in Fig. 1 . The $S_{q \text {-versus- } q \text { curves }}$ exhibit a principal refraction peak as known from other simple liquids [4]. It is caused by the hard-core driven excluded volume phenomenon, $g(r<d)=0$. The hightemperature curves 1 and 2 exhibit peaks, which are only slightly smaller and somewhat broader than the peaks of a HSS at the same densities. The attraction modifies the pair correlations and thus the excluded volume effects, as can be inferred by comparing the curves 1 and 3 . Lowering $T$, the short-ranged attraction causes the particles to move closer, i.e., the most probable interparticle spacing decreases. Therefore, the peak position shifts to higher $q$ upon cooling. The distribution $g(r)$ develops a more rapidly varying structure at distances which are multiples of the particle diameter, and this explains the decrease of the peak height and the increase of the peak wings in $S_{q}$. A change of the density at low temperature modifies the peak in a similar manner as discussed above for large $T$, cf. curves 3 and 4 . However, lowering $\varphi$ drives the system closer to the spinodal, and therefore the exhibited change of $S_{q}$ for small $q$ is larger than expected for a HSS.

Results for the pair correlation $g(r)$ obtained by different closures of Eqs. (4) and (5) and by other methods have been published by Lang et al. [37]. For both a small and a large well width considered there, $\delta=0.03$ and $\delta=0.5$, respectively, we find our results for $g(r)$ in agreement with the Monte Carlo simulation results obtained by Lang et al. Only for the small well width, $g(r)$ is underestimated in the well regime, $1<r<1+\delta$, by about the same amount as Nezbeda's approximation $[39,40]$ overestimates $g(r)$. At $r>1$ $+\delta$, however, our solution appears to be in better agreement 

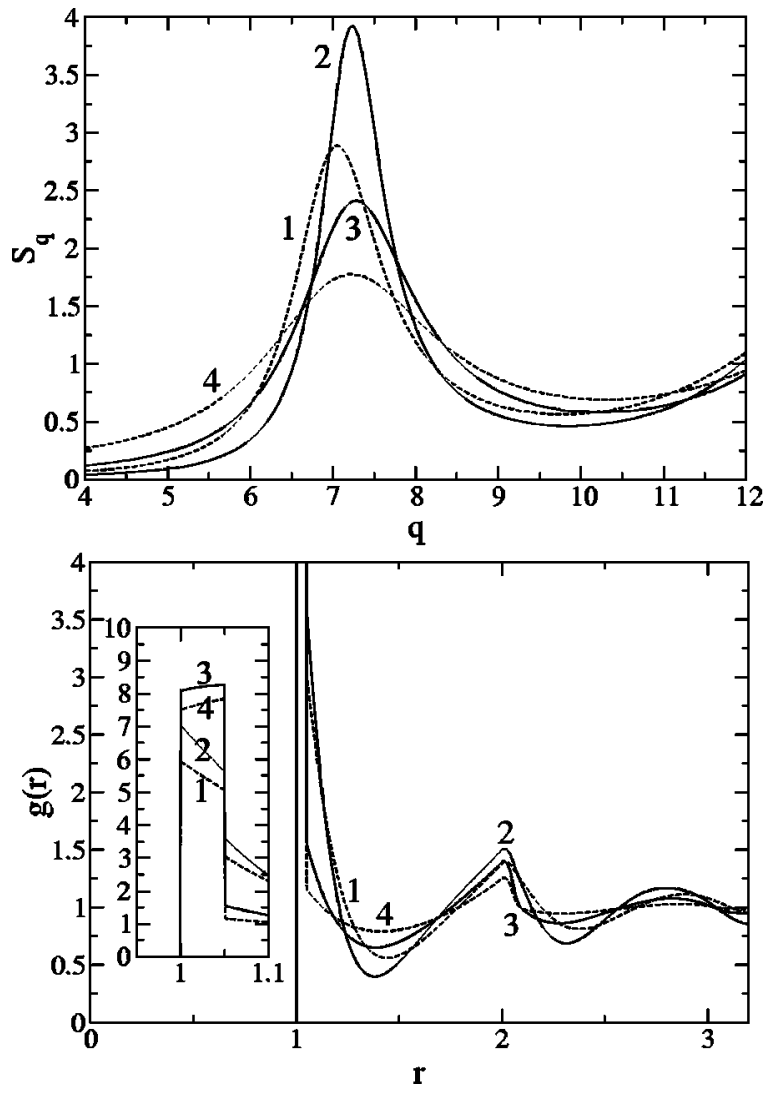

FIG. 2. Structure factor $S_{q}$ and pair correlation function $g(r)$ of the SWS calculated within the MSA for the relative well width $\delta$ $=0.05$. The labels 1 to 4 correspond to the states indicated by the diamonds in Fig. 1. They are given by the pairs $(\varphi, \theta)$ of the packing fraction and reduced temperature $(0.50,0.50),(0.55,0.50)$, $(0.50,0.15)$, and $(0.40,0.15)$, respectively. Here and in the following figures, the hard-core diameter is chosen as the unit of length, $d=1$, and $q$ is given here and in the following in units of $d^{-1}$.

with the simulation results. This behavior is similar to what holds for the optimized random-phase approximation [37].

The large- $q$ tail of $c_{q}$ will be of importance in the following. In Fig. 3 it is shown that the asymptote, Eq. (12), describes $c_{q}$ very well for $q>20$. The results have been evaluated for the state discussed in Fig. 2 with the label 3, where $(A, B, C)=(-0.092,1.63,7)$. The tail consists of a part due to the first two terms in Eq. (12), which differs from the HSS result merely by modifications of the coefficients $A$ and $B$. This part of the asymptote is shown in Fig. 3 (dotted line) in order to emphasize that the last contribution in Eq. (12) can be dominant. The next-to-leading-order contributions to our results are not relevant for a discussion of the qualitative features of the tail. Therefore, let us write the lowest-order formula for the tail as $c_{q}^{\text {as }}=c_{q}^{\text {rep }}+c_{q}^{\text {att }}$. Here, the coefficients of $c_{q}^{\text {rep }}$ are obtained via a Baxter-like limit, $\delta \rightarrow 0, \mathrm{~K}=\delta / \theta$ fixed. Noting $A \rightarrow 0$ in this limit, we find

$$
c_{q}^{\mathrm{rep}}=\left(4 \pi / q^{2}\right) B_{0} \cos (q) .
$$

The attraction-induced tail in this approximation reads

$$
c_{q}^{\mathrm{att}}=\left(4 \pi / q^{2}\right) K(2 / \delta) \sin (q \delta / 2) \sin [q(1+\delta / 2)] .
$$

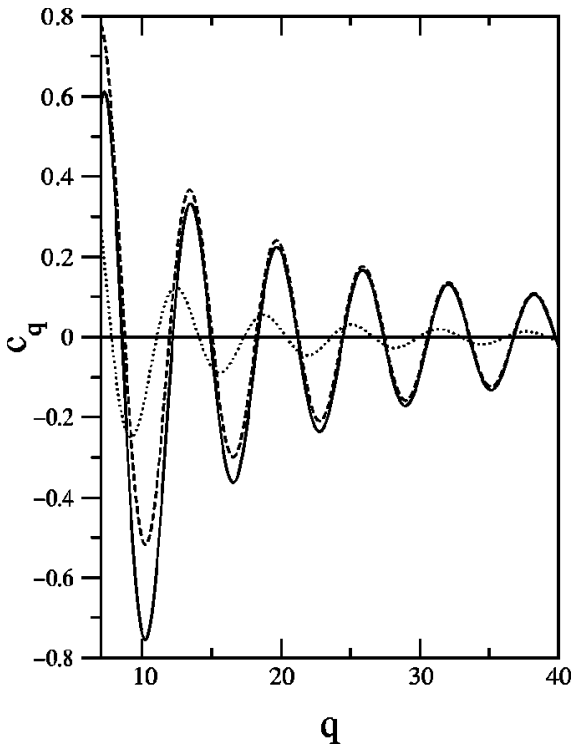

FIG. 3. Direct correlation function $c_{q}$ of the SWS for relative well width $\delta=0.05$ calculated within the MSA (solid line). Density and temperature are the ones considered in Figs. 1 and 2 for the label 3. The dashed line exhibits the leading asymptote $c_{q}^{\text {as }}$ according to Eq. (12). The dotted line represents the same result with coefficient $C$ replaced by zero (see text).

For the example in Fig. 3, $\left(B_{0}, K\right)=(4 / 3,1 / 3)$. For $q$ below an upper cutoff $q_{u}=\pi / \delta$, the function in the bracket of Eq. (14b) increases almost linearly with $q$. This explains the increasing importance of the $c_{q}^{\text {att }}$ contribution relative to the $c_{q}^{\text {rep }}$ one, as is demonstrated in Fig. 3. If $K$ is sufficiently large or if $\left|B_{0}\right|$ is sufficiently small, one can identify a lower cutoff $q_{l}=\left|B_{0}\right| / K$ such that $c_{q}^{\text {att }}$ dominates $c_{q}^{\text {rep }}$,

$$
c_{q}^{\mathrm{as}}=(4 \pi K / q) \sin [q(1+\delta / 2)], \quad q_{l} \ll q \ll q_{u} .
$$

In the wave-vector interval between $q_{l}$ and $q_{u}, c_{q}$ exhibits a power-law decrease slower than the one of the true large- $q$ tail, which dominates only for $q \gg q_{u}$.

The PYA and the MSA differ solely by their ansatz for the direct correlation function $c(r)$ within the attraction shell $1<r<1+\delta$. Within the PYA, $c(r)$ depends on $r$ via the $r$ dependence of the pair distribution function, $c_{\mathrm{PYA}}(r)=\{1$ $\left.-\exp \left[-u_{0} /\left(k_{B} T\right)\right]\right\} g(r)$, while the MSA assumes a constant $c_{\mathrm{MSA}}=u_{0} /\left(k_{B} T\right)$. In this paper, systems with narrow attraction shells are of interest, $\delta \ll 1$. Therefore it is a reasonable approximation to ignore the $r$ dependence of $c_{\mathrm{PYA}}$ by writing $g(r) \approx g_{d}=g(r=1)$. Thus, for every state where a solution of the PYA exists, there is a solution of the MSA, yielding the same structure factor. However, the corresponding solution for the MSA has to be evaluated for an effective reduced temperature $\theta_{\mathrm{eff}}^{\mathrm{MSA}}$. The latter is a smooth function of $\theta$ $=u_{0} / k_{B} T, \varphi$ and $\delta$, estimated by

$$
\frac{1}{\theta_{\mathrm{eff}}^{\mathrm{MSA}}} \approx\left[1-\exp \left(\frac{-u_{0}}{k_{B} T}\right)\right] g_{d} \text {. }
$$




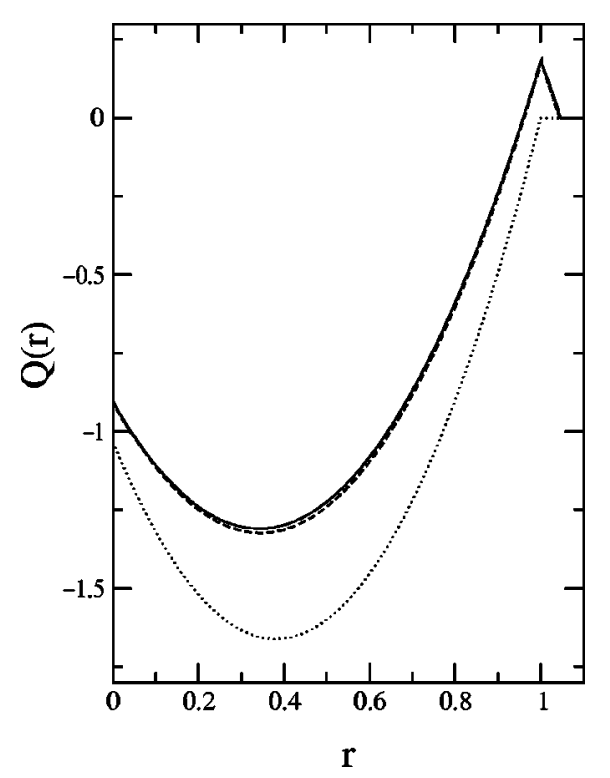

FIG. 4. Factor function $Q(r)$ of the SWS. The hard-core diameter is chosen as the unit of length. The dashed line is the PYA result for $\varphi=0.5293$, reduced temperature $\theta=1.10$, and well-width parameter $\delta=0.0429$. The full line is the MSA result for $\varphi$ $=0.5258, \theta=0.2332, \delta=0.0465$, chosen to represent the same physical state of interest in our discussion; see the text for details. The dotted line shows the result for the HSS at the packing fraction $\varphi=0.516$

Consequently, the PYA and the MSA yield the same scenarios for the structure factor in the parameter regime of interest in this paper. This finding is demonstrated in Fig. 4 for the basic quantity of the structure factor theory, the factor function $Q(r)$. The result calculated within the PYA for the parameter triple

$$
\varphi_{\mathrm{PYA}}^{*}=0.5293, \theta_{\mathrm{PYA}}^{*}=1.1000, \delta_{\mathrm{PYA}}^{*}=0.0429
$$

is very close to the one obtained within the MSA for

$$
\varphi_{\mathrm{MSA}}^{*}=0.5258, \theta_{\mathrm{MSA}}^{*}=0.2332, \delta_{\mathrm{MSA}}^{*}=0.0465 \text {. }
$$

It will be shown in Sec. III that the two states specified above are of central importance. The values found for the corresponding densities $\varphi^{*}$ and well widths $\delta^{*}$ are close to each other. The difference in the values for the effective attraction strength $1 / \theta^{*}$ is well explained by Eq. (15). If one inserts $g_{d}^{*} \approx 7.5$ as obtained from the MSA, one finds $\theta_{\mathrm{eff}}^{\mathrm{MSA}}$ $\approx 0.2233$.

All calculations within the MSA are based on the small- $\delta$ expansion for the factor function, say $Q^{\exp }(r)$, defined by Eqs. (9) to (11). To control this result, Eqs. (6) and the ana$\log$ of Eq. (7) for the MSA closure have also been solved numerically to get the correct MSA factor function, say $Q^{\mathrm{MSA}}(r)$. The difference $\delta Q(r)=Q^{\mathrm{MSA}}(r)-Q^{\exp }(r)$ is positive and about 3\% (1\%) for $\delta=0.25,(0.15)$ for $r \leqslant 0.5$; and it decreases for $r$ increasing above 0.5 .

The dotted line in Fig. 4 exhibits the parabola for $Q(r)$, Eq. (9a), for coefficients of the HSS. Introduction of the attraction smoothly renormalizes the coefficients $a, b$, and $c$, such that the parabola shifts upwards and becomes flatter. There appears a region of positive values for $Q(r)$ near the core surface $r \approx 1$. These shifts cause the smooth drifts of the Fourier transform for $\hat{Q}(q)$, which lead to the drifts of $S_{q}$ discussed in connection with Fig. 2 and to the appearance of a spinodal, cf. Fig. 1. The only qualitative new feature, which is caused by the attraction well, is the almost straight decrease of $Q(r)$ within the interval $1<r<1+\delta$. Equation (9c) yields the slope in leading order as $Q^{\prime}(r)=-K / \delta$. In the Baxter limit, this slope diverges. The specified almost constant part of $Q^{\prime}(r)$ causes the attraction-tail contribution to the asymptote of $c_{q}$, Eq. (14b). The power-law tail, Eq. (14c), is a precursor of the mentioned divergency.

The structure factor or the pair correlation function determine the positions of the liquid-gas transition points. However, one faces the known consistency problem that different routes for the equation of state yield different results for the transition points if approximations for $S_{q}$ or $g(r)$ are used [4]. We will not discuss these problems in this paper, since it is irrelevant for the evolution of glassy dynamics or the glass transition whether the fluid is in a stable or metastable thermodynamic state.

\section{PHASE DIAGRAM}

\section{A. The bifurcation equation}

The MCT equations of motion for various dynamical quantities are based on the equations for the normalized density correlators $\phi_{q}(t)=\left\langle\rho_{q}^{*}(t) \rho_{q}^{-}\right\rangle /\left\langle\left|\rho_{q}^{-}\right|^{2}\right\rangle$. For the liquid state these functions approach zero for large time $t$; density fluctuations which were created at time $t=0$ disappear for $t \rightarrow \infty$. The glass state is characterized by a spontaneous arrest of these fluctuations, i.e., the long-time limits $f_{q}$ of the correlators do not vanish. The ideal liquid-glass transition of the MCT is characterized by a discontinuous increase of $f_{q}$ from its value zero in the liquid state to the critical DebyeWaller factor $f_{q}^{c}>0$ of the glass. For colloidal suspensions, $f_{q}$ can be deduced from the dynamical light-scattering results for $\phi_{q}(t)$. The experimental findings for the HSS [10] and for a charge stabilized system [23] confirmed the discontinuity for $f_{q}$ and the data for $f_{q}^{c}$ agree well with the MCT results.

The $f_{q}$ obey the equation $f_{q} /\left(1-f_{q}\right)=\mathcal{F}_{q}(f)$ [14]. Here, the mode-coupling functional $\mathcal{F}_{q}$ is given by

$$
\mathcal{F}_{q}(f)=\frac{1}{2} \int \frac{d^{3} k}{(2 \pi)^{3}} V_{\vec{q}, \vec{k}} f_{k} f_{|\vec{q}-\vec{k}|} .
$$

The mode-coupling vertices are determined by the structure factor $S_{q}$, the direct correlation function $c_{q}$, and the density $\rho$,

$$
V_{\vec{q}, \vec{k}} \equiv S_{q} S_{k} S_{|\vec{q}-\vec{k}|} \rho\left[\vec{q} \cdot \vec{k} c_{k}+\vec{q} \cdot(\vec{q}-\vec{k}) c_{|\vec{q}-\vec{k}|}\right]^{2} / q^{4} .
$$

In the following, the wave-vector integrals will be discretized to points on a grid of $M$ values, which are equally 
spaced with step size $h$, starting at $q_{\min }=h / 2$. Thereby the mode-coupling functional is changed to a second-order polynomial

$$
\mathcal{F}_{q}(f)=\sum_{k p} V_{q, k p} f_{k} f_{p}
$$

The explicit representation of the coefficients can be found in Ref. [41]. The $M$ parameters $f_{q}$ obey the algebraic equations

$$
f_{q} /\left(1-f_{q}\right)=\mathcal{F}_{q}(f), \quad q=1, \ldots, M .
$$

Besides the long-time limit $f_{q}$, Eq. (19) can have further solutions, say $\widetilde{f}_{q}$, obeying $0 \leqslant \widetilde{f}_{q}<1$. The Debye-Waller factor is distinguished by the maximum property $f_{q} \geqslant \widetilde{f}_{q}, q$ $=1, \ldots, M[42]$. We used the iteration procedure $f_{q}^{(n+1)}$ $=\mathcal{F}_{q}\left[f^{(n)}\right] /\left(1+\mathcal{F}_{q}\left[f^{(n)}\right]\right), \quad n=0,1, \ldots$ to determine $f_{q}$. With increasing $n$ the $f_{q}^{(n)}$ decrease monotonically towards $f_{q}$, if the iteration is started with $f_{q}^{(0)}=1$ [43].

Two concepts are needed in the following, namely the maximum eigenvalue $E$ and the exponent parameter $\lambda$ [42]. For the discussion of the implicit equations, Eq. (19), the Jacobian $J$ is of importance. It is equivalent to $1-C$, where the $M \times M$ matrix $C$ is determined by

$$
C_{q k}=\frac{\partial \mathcal{F}_{q}(f)}{\partial f_{k}}\left(1-f_{k}\right)^{2} .
$$

Also the variation of $C$ with changes of $f$ is needed

$$
C_{q, k p}=\frac{1}{2} \frac{\partial^{2} \mathcal{F}_{q}(f)}{\partial f_{k} \partial f_{p}}\left(1-f_{k}\right)^{2}\left(1-f_{p}\right)^{2} .
$$

There is a nondegenerate eigenvalue $E$ of matrix $C$ with the property that all other eigenvalues $\widetilde{E}$ obey $\widetilde{E}<E$. There holds $E \leqslant 1$, and liquid-glass transition points are determined by the condition $E=E_{c}=1$. It is helpful to follow the drift of $E$ with changes of control parameters while searching for the transition points. The left and right eigenvectors of $C$ for the eigenvalue $E$, denoted by $\hat{e}$ and $e$, respectively, are uniquely determined by the conditions: $\hat{e}_{q} \geqslant 0, e_{q} \geqslant 0, \Sigma_{q} \hat{e}_{q} e_{q}$ $=1, \Sigma_{q} \hat{e}_{q}\left(1-f_{q}\right) e_{q}^{2}=1$. They are used to characterize every transition point by a single number $\lambda$, defined as

$$
\lambda=\sum_{q k p} \hat{e}_{q}^{c} C_{q, k p}^{c} e_{k}^{c} e_{p}^{c}
$$

The solutions of Eq. (19), considered as functions of the $M^{3}$ coefficients $V_{q, k p}$, can exhibit singularities, which are called bifurcation points [44]. The singularities occur if the Jacobian $J$ is a singular matrix, i.e., if the matrix $C$ has eigenvalue unity. The special singularities, which are exhibited by the Debye-Waller factors, are called glass-transition singularities. These are members of the simplest family of singularities, labeled $A_{l}, l=2,3, \ldots$ [44]. They are topologically equivalent to the bifurcation singularities of the real roots of real polynomials of degree $l$. Since the $V_{q, k p}$ are smooth functions of the control parameters, in the SWS the
Debye-Waller factor $f_{q}$ exhibits $A_{l}$ singularities considered as a function of the variables $\varphi, \theta$, and $\delta$. The liquid-glass transition is an example for the simplest bifurcation singularity $A_{2}$, called a fold bifurcation. Such transitions occur on smooth surfaces in the three-dimensional parameter space. These surfaces can terminate in smooth lines of $A_{3}$ singularities, which are also called cusp bifurcations. The inner points of the $A_{2}$ surfaces are characterized by $0<\lambda<1$, and for the end points there holds $\lambda=1$. The most complicated generic singularity in a three-parameter system is the meeting of two $A_{3}$ lines in an $A_{4}$ point. Its position shall be denoted by $\varphi^{*}, \theta^{*}$, and $\delta^{*}$. This singularity is also called a swallow-tail bifurcation [44]. The possibility of the described scenarios has been demonstrated earlier for schematic MCT models, invented with the mere intention of demonstrating the existence of $A_{3}$ and $A_{4}$ points [42]. This paper is the first demonstration of the existence of an $A_{4}$ for a microscopic model; the values for the SWS are given in Eqs. (16).

The numerical work is done with step size $h d=0.4$. It was checked for representative cases, that choosing smaller step sizes does not alter the results to be presented. Choosing $M$ is equivalent to introducing an upper wave-vector cutoff $q^{*}=(M-1 / 2) h$ in Eq. (17a). The previous comprehensive studies for the HSS [41,45] were done with $M=100$. For sufficiently large $1 / \theta$ and sufficiently small $\delta$, the direct correlation function $c_{q}$ develops a large- $q$ tail, discussed in Eqs. (14). This is decisive for fixing the value $q^{*}$ needed to ensure the correct handling of Eqs. (17). If the coefficient $K$ in Eq. (14c) is kept fixed, the cutoff $q^{*}$ will increase with the decreasing well-width parameter proportional to $1 / \delta$. The maximum value for $M$ that can be handled in the numerical work defines the lower limit for $1 / K$ and $\delta$, which can be treated. We used values for $M$ up to 2000 occasionally, in order to guarantee the cutoff independence of the results reported in this paper.

\section{B. Results}

The phase diagram for the SWS is shown in Fig. 5 for several constant- $\delta$ cuts through the three-dimensional control-parameter space. The results based on the PYA and the MSA are qualitatively the same. Let us first consider the three states 1,2 , and 3 from Fig. 1 for $\delta=0.06$. Within the MSA, state 1 refers to the liquid phase, cf. Fig. 5(b). Increasing $\varphi$ to the state 2 increases the height of the first sharp diffraction peak of $S_{q}$, located near $q_{0}=7$, cf. Fig. 2. Thus the compressibility for fluctuations in the shell $q \approx q_{0}$ increases. This leads to arrest in a glass state, as known from the HSS. If one cools state 1 at fixed $\varphi=0.50$ down to state $3, S_{q_{0}}$ decreases, as was explained in connection with Fig. 2. This effect stabilizes the liquid, but it is overcompensated by the increase of $S_{q}$ in the small-wave-vector region, $q<6.1$, and, more important, in the large- $q$ region, $q>7.4$. As a result of this compressibility increase on the wings of the structure factor peak, the liquid freezes to a glass upon cooling, cf. Fig. 5. For large temperature, $S_{q}$ depends only weakly on $T$; the terms proportional to $K=\delta u_{0} /\left(k_{B} T\right)$ in Eqs. (9) to (11) cause only small modifications of the coefficients determining the factor function $Q(r)$. This explains 

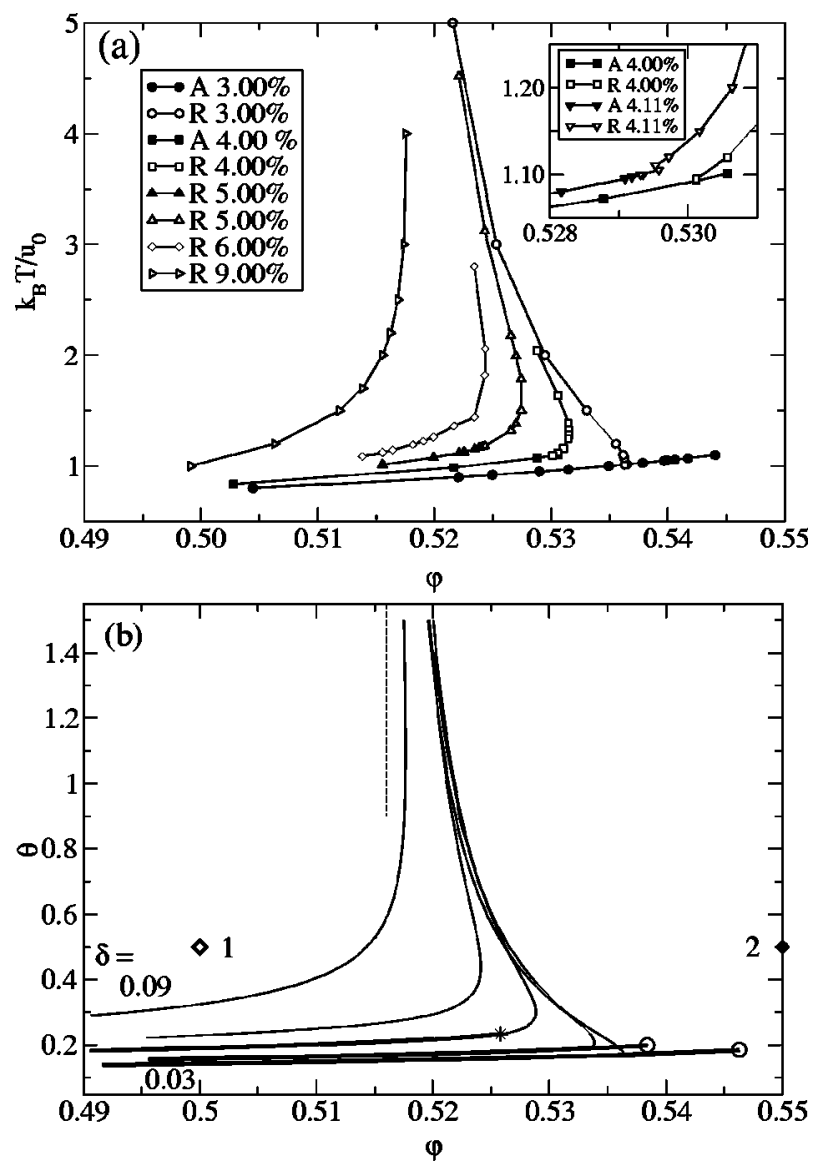

FIG. 5. The phase diagram of the SWS showing cuts through the control parameter space for fixed relative attraction-well width $\delta=\Delta / d$. The upper part (a) is based on the PYA for the structure factor $S_{q}$, and the ratio $\delta /(1+\delta)$ is noted in the legend. The lower part (b) is based on the MSA for $S_{q}$, and the well widths are $\delta$ $=0.09,0.06,0.0465,0.035$, and 0.03, subsequently. The $A_{3}$ end points are marked by open circles and the $A_{4}$ by an asterisk. The vertical dashed line marks the transition line $\varphi \approx 0.516$ for the hardsphere system. For reference, states 1 and 2 from Fig. 1 are included as diamonds.

why the transition lines are almost vertical in Fig. 5(a) for $k_{B} T / u_{0}>3$ and in Fig. 5(b) for $\theta>1$. The peak wings are not very sensitive to density changes by a few percent for $\varphi$ $<0.51$. This explains why the transition lines in Fig. 5 are rather flat there. The two pieces of the transition line join smoothly and $\lambda$ remains below unity for $\delta=0.06$, as is shown in Fig. 6(b). Thus, the described curve represents a cut through a smooth surface of $A_{2}$ bifurcations.

The mentioned high-temperature pieces of the transition surface are located at packing fractions $\varphi_{c}$, which exceed the value for the HSS, $\varphi_{c}^{\mathrm{HSS}} \approx 0.516$. This means that the attraction forces have stabilized the liquid phase. This effect is smaller for larger $T$ and therefore the $\varphi_{c}$-versus- $T_{c}$ curve decreases. There is the possibility of glass melting due to cooling, if the decrease of $S_{q_{0}}$ is not overcompensated by the increase of the structure-factor-peak wings. The attraction causes bonding, in the sense that the average separation of two particles is smaller than expected for a HSS. Therefore the average size of the holes increases and this favors the
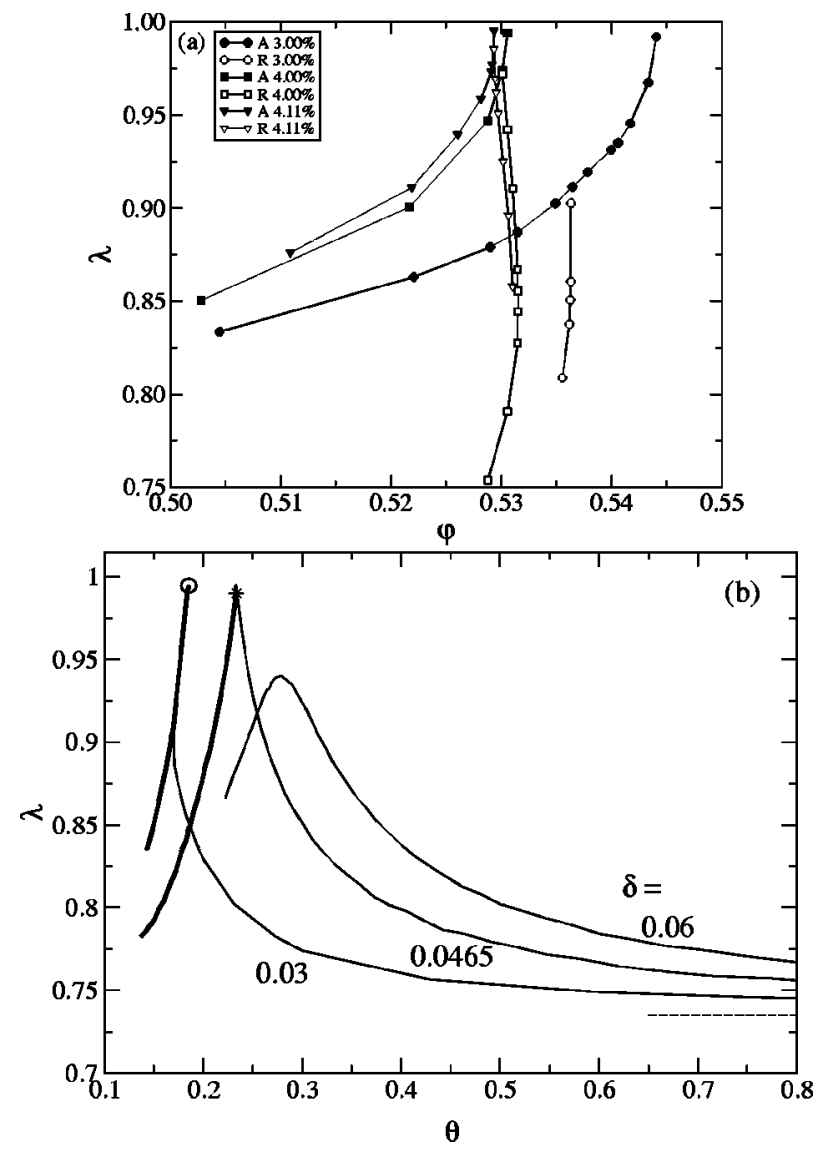

FIG. 6. Exponent parameter $\lambda$ for points on three transition lines. The upper part (a) was calculated within the PYA for the ratios $\delta /(1+\delta)$ noted in the legend. Part (b) shows the results for the MSA, where the dashed line indicates the value $\lambda=0.735$ of the HSS.

long-distance motion characteristic for a liquid. Consider states with $\varphi=0.52$ and $\delta=0.06$. For the MSA results one notices from Fig. 5(b) that the system is in a glass for $\theta$ $=0.10$ and it melts upon heating if $\theta$ approaches $\theta_{m}^{-}$ $\approx 0.30$. This transition occurs due to the temperature drift of the coupling to modes with wave vectors in the wings of the $S_{q}$ peak. The system remains in the liquid upon further heating until it reenters the glass at $\theta_{m}^{+} \approx 1.41$. This freezing is caused by the drift of the coupling to modes with $q \approx q_{0}$. The described reentry phenomenon $[24,25]$ is a manifestation of two mechanisms for localization due to the cage effect in the high-density SWS to be explained below.

The preceding two paragraphs can be summarized as follows. There is a subtle interplay of excluded-volume and bonding effects which determine the variations of the pair correlation function $g(r)$ on the length scale of the particle diameter $d$. This is reflected in the properties of the structure factor $S_{q}$ for wave numbers $q$ at and around the peak position $q_{0}$; the relevant $q$ range is the one exhibited in Fig. 2. Fluctuations with longer wavelength are of no qualitative importance for those parameter points studied in this paper. This conclusion was corroborated by dropping all contributions to the mode-coupling functional, Eq. (18), where $k$ or $p$ are smaller than 4; there was no significant change of the phase 
diagram calculated with the MSA structure compared to what is shown in Fig. 5(b). Similarly, a cutoff $q_{+}=20$ was introduced such that all contributions to the mode-coupling functional with $q>q_{+}$are dropped. The MSA results shown in Fig. 5(b) for $\delta=0.09$ and $\delta=0.06$ did not change, nor was there a noticeable change for the other curves for $\theta>0.6$. We conclude that the two specified sources for correlations on the intermediate length scale explain the phase transition points, which are marked by open symbols in Fig. 5(a) or by the corresponding light lines in Fig. 5(b).

To substantiate the previous conclusion, we have constructed a further phase diagram based on the MSA structure factor, using the above specified cutoff $q_{+}$. As mentioned, the curves for $\delta=0.09$ and $\delta=0.06$ were reproduced up to minor deviations. Upon decreasing $\delta$ further, curves emerge, which continue the trend of the two curves for larger $\delta$. The limit $\delta \rightarrow 0$ can be carried out; no new features appear. This ad hoc MCT model yields a smooth liquid-glass transition surface of $A_{2}$ bifurcation points with $\lambda<1$. Obviously, the true phase diagram of the SWS shown in Fig. 5 is quite different. There are the transition points marked by filled symbols in Fig. 5(a) or by the corresponding heavy lines in Fig. 5(b). These define transition lines, which do not join smoothly the previously discussed lines. Rather they cross the former lines. Thus, for sufficiently small $\delta$ and sufficiently large attraction strength $u_{0} /\left(k_{B} T\right)$, there is a new glass formation mechanism, dominated by density fluctuations with large wave number $q \geqslant q_{+}$. These are due to spatial correlations on the length scale of the attraction-well width $\Delta$.

For a discussion of the identified new pieces of the transition surface, the mode-coupling coefficients in Eqs. (17a) and (17b) can be simplified. As explained in connection with Fig. 3, one can write for $k=|\vec{k}| \geqslant q_{+}$and $p=|\vec{q}-\vec{k}| \geqslant q_{+}$the leading asymptotic expression for the structure factors $S_{k}$ $=S_{q}=1$, and for the direct correlations function $c_{q}=c_{q}^{\text {as }}$, Eq. (14b). Thus, the dominant part of the mode-coupling functional depends explicitly on the control parameters by the prefactor $\varrho K^{2}=\varrho\left[\delta u_{0} /\left(k_{B} T\right)\right]^{2}$ and otherwise only on $\delta$ via the large-wave-vector cutoff $q_{u}$. A further density dependence is due to fluctuations with $q<q_{+}$only. This explains why the low- $T$ transition lines in Fig. 5 are so flat. $K^{2}$ decreases proportionally to $\delta^{2}$, and this effect is not overcompensated by the increase of $q_{u}$. As a result, the horizontal transition lines decrease with decreasing $\delta$. For low packing fractions, such a trend can be shown explicitly by an analytic calculation $[25,46]$. Along this transition line, $\lambda$ increases with increasing $\varphi$ until it approaches unity signalizing an end point at some $\varphi_{c}^{\circ}(\delta), \theta_{c}^{\circ}(\delta)$. This is demonstrated in Fig. 6(a) for three values of $\delta$ and in Fig. 6(b) for $\delta=0.03$. The line stops the previously discussed line in some crossing point. Between the crossing point and the end point, there occur glass-glass transitions. Upon increasing $\delta$, the length of the glass-glass transition line shrinks, until it vanishes for some $\delta^{*}$ at some $\varphi^{*}=\varphi_{c}^{\circ}\left(\delta^{*}\right)$ and $\theta^{*}=\theta_{c}^{\circ}\left(\delta^{*}\right)$; and this is the $A_{4}$ singularity whose coordinates are listed in Eqs. (16).

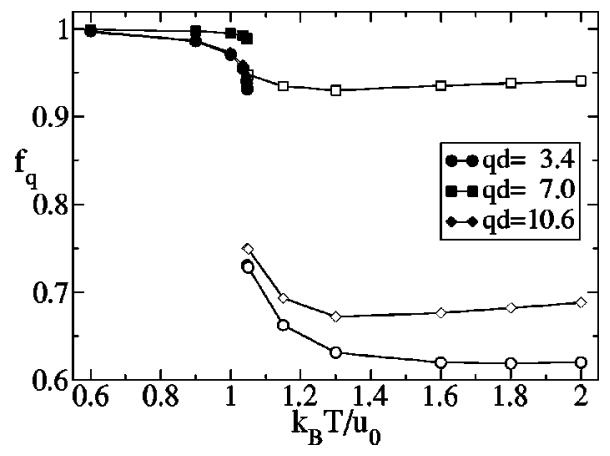

FIG. 7. The Debye-Waller factor $f_{q}$ as a function of the reduced temperature for the fixed packing fraction $\varphi=0.539672$ and fixed $\delta /(1+\delta)=0.03$. The wave vector $q d=7.0$ is close to the structure factor peak position. The calculations are based on the PYA for $S_{q}$. The path through the parameter space deals with a glass-glass transition occuring at $\theta_{c}=1.0471$, compare Fig. 5(a).

Every pair of glass states can be connected by a curve in parameter space such that, upon shifting the control parameters $\varphi, \theta$, and $\delta$ along this curve, the glass properties change smoothly. Thus one cannot discriminate precisely between repulsion- and attraction-dominated glass states. However, upon crossing the glass-glass-transition surface, there occurs a discontinuous change of the glass-state properties. These discontinuities can be used to differentiate quantitatively between the two types of glasses. From a mathematical point of view, the situation is analogous to the termination of the liquid-gas-transition line at the critical point. Some results shall be presented from the theory based on the PYA for $S_{q}$. Let us consider states for $\varphi \approx 0.54$ for the smallest $\delta$ used in Fig. 5(a). Figure 7 exhibits as filled symbols the decrease of the Debye-Waller factor for three representative wave numbers, if the attraction-dominated glass is heated towards the transition temperature $T_{c}, k_{B} T_{c} / u_{0}$ $=1.0471=\theta_{c}$. The $f_{q}$ decrease towards the critical value $f_{q}^{c}$ according to the asymptotic square-root law $f_{q}-f_{q}^{c}$ $\propto h_{q} \sqrt{\theta_{c}-\theta}$, which is the signature of the fold bifurcation [42]. Upon crossing the line into the repulsion-dominated glass, $f_{q}$ drops and keeps on decreasing upon further heating up to $\theta \approx 1.3$, as shown by the open symbols. Notice that $f_{q}$ does not exhibit any singularity for $\theta$ decreasing towards $\theta_{c}$. The remarkable variation of $f_{q}$ for $\theta$ near but above $\theta_{c}$ is a precursor phenomenon of the nearby $A_{3}$ singularity. The $f_{q}$ for $\theta>\theta_{c}$ is smaller than the Debye-Waller factor for the HSS at the same packing fraction. Hence the attraction has softened the glass. This effect has to disappear for very large $T$, and this explains why $f_{q}$ increases again, reflecting a glass stiffening upon heating. The described effects for $\theta>\theta_{c}$ are the counterparts to what was discussed above in connection with the reentry phenomenon.

The wave-vector dependence of $f_{q}$ changes qualitatively upon crossing the glass-glass-transition line as is shown in Fig. 8. Let us focus in this paragraph on the wave-vector regime at and above the structure factor peak position $q$ $\gtrsim q_{0} \approx 7$. Here, $f_{q}$ oscillates with wave-vector scale $2 \pi / d$ around the Mößbauer-Lamb factor $f_{q}^{s}$. The latter is the ana$\log$ to $f_{q}$, constructed for a tagged particle with position 


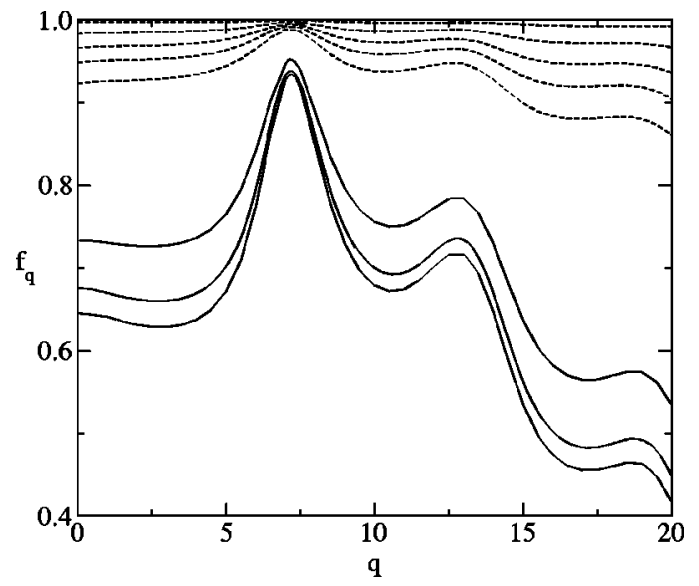

FIG. 8. The Debye-Waller factors $f_{q}$ for $\varphi=0.539672, \delta /(1$ $+\delta)=0.03$. The temperature increases from top to bottom as $\theta$ $=k_{B} T / u_{0}=0.600,0.900,1.000,1.035,1.0471$ (dashed lines) and $\theta$ $=1.047,1.150,1.300$ (full lines). The calculations are based on the PYA for $S_{q}$.

vector $\vec{r}_{s}(t)$ via its density-correlator $\phi_{q}^{s}(t)=\left\langle\rho_{\vec{q}}^{s}(t)^{*} \rho_{\vec{q}}^{s}\right\rangle$; $\rho_{\vec{q}}^{s}(t)=\exp \left[i \overrightarrow{i q} \vec{r}_{s}(t)\right]$. This quantity, in particular its long-time limit $f_{q}^{s}$, can also be measured [13]. One finds $f_{q}^{s}=1$ $-q^{2} r_{l}^{2}+\mathcal{O}\left(q^{4}\right)$, where $r_{l}$ is the localization length of the particle: $r_{l}^{2}=\lim _{t \rightarrow \infty}\left\langle\left|\vec{r}_{s}(t)-\vec{r}_{s}(0)\right|^{2}\right\rangle$ [42]. In the Gaussian approximation, one can write $f_{q}^{s}=\exp \left(-q^{2} r_{l}^{2}\right)$ so that the half-width wave vector $q_{l}$, defined by $f_{q_{l}}=0.5$, can be used to estimate $r_{l} \approx 1 / q_{l}$. For the Debye-Waller factors on the high-temperature side of the transition line, which are shown as full lines in Fig. 8, one estimates $q_{l} \approx 20$. The localization length $r_{l}=0.05$ is about the same size as expected for a particle rattling between the hard walls of its cage in a HSS. The $f_{q}$ and $r_{l}$ are close to those of a HSS $[42,45]$. However, on the low-temperature side of the transition, the $f_{q}$ vary by less than $10 \%$ if $q$ increases up to $2 q_{0}$, as shown by the dashed lines in Fig. 8. The wave number $q_{l}$ is much larger than expected from the free volume in the cage. At the transition, $q_{l} \approx 44$, i.e., the localization length is decreased discontinuously by a factor of about 2.3 . For $\theta=0.9$ and 0.6 the localization length is about 0.01 and 0.006 , respectively. This shows that the localization length is of order $\delta$. The particle is bound to the wall of the cage and localization is determined entirely by the particle attraction. This localization mechanism via bond formation is operative at low packing fractions also, and it has been studied within the MCT in this regime. There, bond formation has been argued to be of importance for colloidal gelation [25,46,47].

There is no strong dependence of $f_{q}$ on wave numbers $q$ $<4$. The low-temperature glass is distinguished from the high-temperature one by the fact that the zero-wave-number limit of the Debye-Waller factor, $f_{0}$, is larger for the former than for the latter. Therefore, upon crossing the glass-glasstransition surface by cooling, the peak for $q \approx q_{0}$ of the $f_{q}$-versus- $q$ diagram disappears, Fig. 8 . The number $f_{0}$ is related to the longitudinal elastic modulus of the system. This consists of a part expected for the ergodic liquid and a part $m_{0}$ reflecting the incomplete relaxation of the noner-

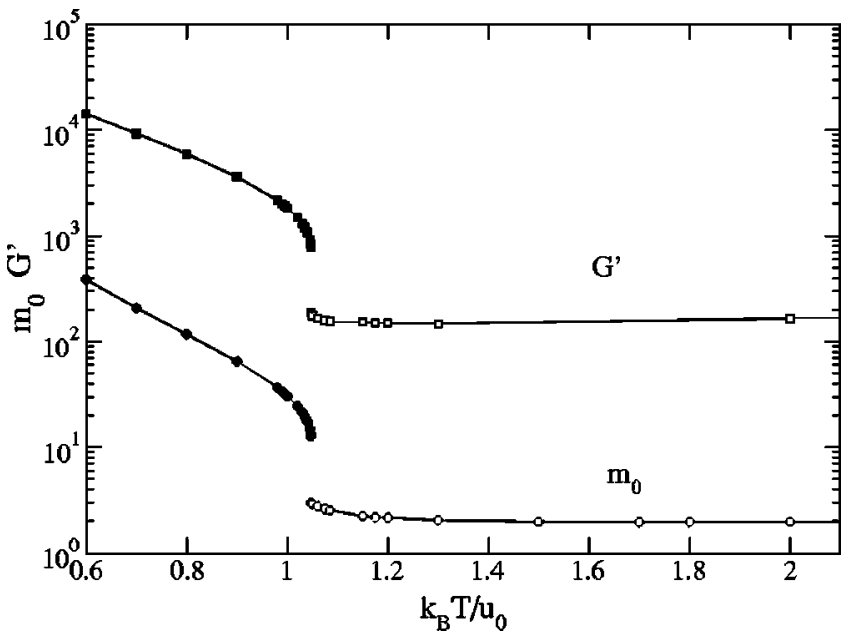

FIG. 9. The shear modulus $G^{\prime}$ and the dimensionless longitudinal elastic modulus $m_{0}$ as a function of the reduced temperature. The result is based on the PYA for $S_{q}$, and the parameters of state deal with the same path through the glass-glass transition as discussed in Figs. 7 and 8.

godic glass [42]. The latter is given by the zero-wavenumber limit of the mode-coupling functional, Eq. (18), $m_{0}$ $=\mathcal{F}_{0}(f)$. The $q=0$ limit can be carried out easily in Eq. (17b), so that one derives from Eq. (17a) a formula [14]

$$
m_{0}=\int_{0}^{\infty} d k v^{L}(k) f_{k}^{2}
$$

After discretization, one can substitute the results for $f_{k}$ to get $m_{0}$, and via Eq. (19) one has $f_{0}=m_{0} /\left(1+m_{0}\right)$. For the glass with $\theta>\theta_{c}$, the integral in Eq. (22) is dominated by fluctuations on the intermediate wave-vector scale, $k \approx q_{0}$. As known from the HSS, $m_{0}$ is of order unity and thus $f_{0}$ is about 0.5 . However, for $\theta \leqslant \theta_{c}$, the integral is dominated by large- $k$ fluctuations. This enhances $m_{0}$. For $\theta=\theta_{c}$, one finds $m_{0} \approx 12.7$ and this increase of the modulus explains the increase of $f_{0}$ to about 0.927 , exhibited by the lowest dashed line in Fig. 8. Decreasing the temperature to $k_{B} T / u_{0}=0.6$ leads to $m_{0} \approx 385$ and this explains the large value $f_{0}$ $\approx 0.997$, exhibited by the uppermost dashed curve in Fig. 8 . For the shear modulus $G^{\prime}$, a formula like Eq. (22) holds, where the expression for $v^{T}$ is similar to that for $v^{L}$ [14]. Therefore, the shear modulus also exhibits the specified strong enhancement due to the attraction wells. Figure 9 shows that the dramatic change of the moduli is the most relevant feature to be observed upon crossing the glass-glasstransition surface. The strong short-ranged attraction causes bond formation, and this increases the rigidity of the glass with respect to compressions or shearing considerably relative to that of a glass at a similar density, where the structural arrest is dominated by mere hard-sphere repulsion.

\section{STRUCTURAL RELAXATION}

\section{A. Some general MCT equations}

The MCT equations of motion are based on the exact expression of the density correlator $\phi_{q}(t)$ in terms of a 
fluctuating-force correlator [4,5], which in turn is split into a part treating normal liquid effects, and a relaxation kernel $m_{q}(t)$ describing the cage-effect contribution. This kernel is approximated by the mode-coupling functional, discussed above in connection with Eqs. (17) and (18): $m_{q}(t)$ $=\mathcal{F}_{q}[\phi(t)][14,42]$. This paper will be restricted to the simplest approximation for the normal liquid effects, i.e., the colloid will be treated as a system of Brownian particles, so that only the instantaneous correlations as given by the structure factor $S_{q}$ are incorporated. As a result one obtains [41]

$$
\tau_{q} \partial_{t} \phi_{q}(t)+\phi_{q}(t)+\int_{0}^{t} m_{q}\left(t-t^{\prime}\right) \partial_{t^{\prime}} \phi_{q}\left(t^{\prime}\right) d t^{\prime}=0 .
$$

This equation implies the short-time asymptote $\phi_{q}(t)=1$ $-\left(t / \tau_{q}\right)+\mathcal{O}\left(t^{2}\right)$. For the time scale, one finds $\tau_{q}$ $=S_{q} /\left(D_{0} q^{2}\right)$, where $D_{0}$ denotes the single-particle diffusion coefficient. $D_{0}$ reflects the property of the solvent, and it fixes the time scale for the transient motion. The unit of time shall be chosen such that $1 / D_{0}=160$ to ease comparisons with the results for the HSS from preceding work $[41,45]$.

Two comments on the implications of Eq. (23) might be appropriate. First, the solutions are completely monotone functions, i.e., there is a rate density $\rho_{q}(\gamma) \geqslant 0$, normalized to $\int_{0}^{\infty} \rho_{q}(\gamma) d \gamma=1$, such that

$$
\phi_{q}(t)=\int_{0}^{\infty} e^{-\gamma t} \rho_{q}(\gamma) d \gamma
$$

Thus, the MCT approximations maintain a fundamental property of colloidal dynamics: autocorrelation functions can be written as superpositions of Debye-relaxation functions [43]. Second, outside the transient, the solutions can be written as $\phi_{q}(t)=F_{q}\left(t / t_{0}\right)$. Here, $F_{q}$ is given by the modecoupling functional $\mathcal{F}_{q}$, i.e., by the equilibrium structure factor $S_{q}$. The transient dynamics, no matter how complicated, enters via the single time scale $t_{0}$ only $[16,48,49]$. The following results for the long-time dynamics are thus not influenced by the simplified treatment of the short-time dynamics in Eq. (23), except up to a change of the overall time scale $t_{0}$. It is known that the short-time dynamics in colloids is influenced by hydrodynamic interactions [2]. Unfortunately, it is not known how to incorporate these interactions in a theory for high-density colloids. But we consider it plausible, that the hydrodynamic interactions merely renormalize the transient dynamics [50], thereby being irrelevant for the structural-relaxation effects.

For control parameters approaching a glass-transition singularity, there appears an increasingly larger dynamical window, where the solutions are arbitrarily close to the critical Debye-Waller factor $f_{q}^{c}$. Therefore, one can solve the MCT equations of motion by an asymptotic expansion, using $\delta \phi_{q}(t)=\phi_{q}(t)-f_{q}^{c}$ as a small parameter. The result can be expressed in the form

$$
\phi_{q}(t)-f_{q}^{c}=h_{q} G(t)+h_{q}^{(1)} G^{(1)}(t)+\cdots .
$$

Most of the known universal results for the MCT bifurcation dynamics are based on the understanding of the leadingorder contribution $G(t)$. The next-to-leading-order term $G^{(1)}(t)$ allows one to discuss the range of validity of the leading-order formulas. A comprehensive demonstration of the cited results for the HSS can be found in Refs. [41] and [45].

Equation (25) reduces to a particular transparent form for the critical dynamics, i.e., for control parameters placed on the glass-transition points. For the fold bifurcation, one finds a power-law decay

$$
G(t)=\left(t_{0} / t\right)^{a}, G^{(1)}(t)=\left(t_{0} / t\right)^{2 a} .
$$

The critical exponent $a$ is given by the exponent parameter $\lambda$ via $\Gamma(1-a)^{2} / \Gamma(1-2 a)=\lambda, 0<a \leqslant 0.5$. The end points of the $A_{2}$ bifurcation surfaces are characterized by exponent $a$ approaching zero. For the cusp bifurcation, there holds [51]

$$
\begin{gathered}
G(t) \propto 1 / \ln \left(t / t_{0}\right)^{2}, \\
G^{(1)}(t) \propto \ln \left[\ln \left(t / t_{0}\right)\right] / \ln \left(t / t_{0}\right)^{3} .
\end{gathered}
$$

For the swallow-tail bifurcation, one has $[51,52]$ for $A_{4}$

$$
\begin{gathered}
G(t) \propto 1 / \ln \left(t / t_{0}\right), \\
G^{(1)}(t) \propto \ln \left[\ln \left(t / t_{0}\right)\right] / \ln \left(t / t_{0}\right)^{2} .
\end{gathered}
$$

The dependence of the leading-order contribution $G(t)$ on the control parameters is well understood, but shall not be considered in this paper.

\section{B. Results}

Let us first estimate the dynamical window relevant for the discussion. For the HSS, the correlators $\phi_{q}(t)$ decay from 1.00 to 0.95 for times increasing up to about $t=1$ for representative wave vectors. In this sense, $t=1$ is the scale for the transient dynamics. After a crossover window of about one or two decades, the leading-order asymptotic law $\phi_{q}(t)=f_{q}^{c}+h_{q} G(t)$ becomes valid at about $t=10^{2}$. This value may be an order of magnitude smaller or larger, depending on the wave number $q$ [41]. The same is true for the data obtained by van Megen et al. for hard-sphere colloids, provided one identifies the time unit $t=1$ with $1 \mathrm{msec}$ [17]. The correlators have been measured up to $10^{6} \mathrm{msec}$, and thus the so far explored windows extend up to $10^{6}$ in the units used here. This limit might shift up in future work, using different experimental setups.

Figure 10 exhibits the critical correlators $\phi_{q}^{c}(t)$ for $q$ $=4.2$ for five states on the transition line $\delta=0.0465$ through the $A_{4}$ singularity. The states 1 and 5 refer to an exponent parameter $\lambda \approx 0.80$ on the side of the attraction-dominated and repulsion-dominated glass, respectively. For times of the order of $10^{3}$ and larger, the leading-order formula, $\phi_{q}(t)$ $-f_{q}^{c} \propto\left(t_{0} / t\right)^{a}, a \approx 0.28$, describes the results. Thus, the scenario is similar to the one known from the HSS, and this is also true for other states on the line with $\lambda<0.80$. However, 


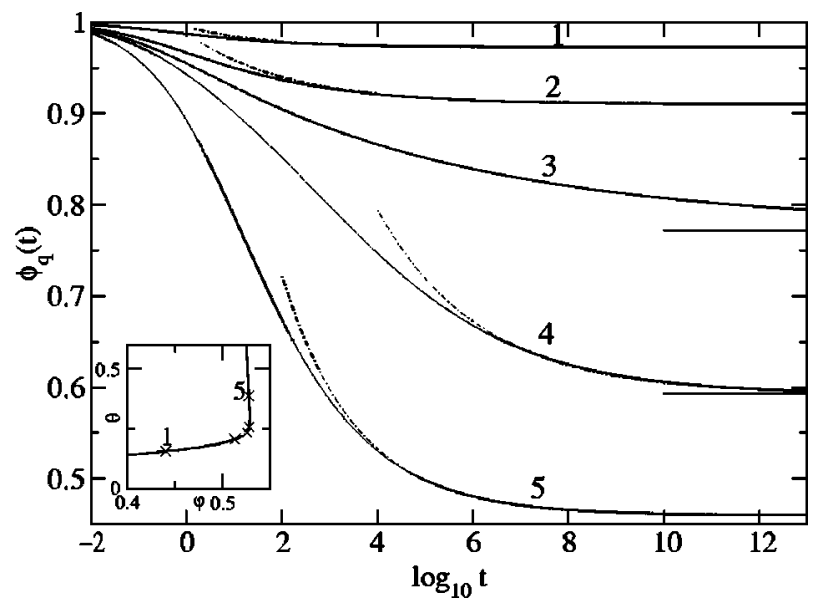

FIG. 10. The full lines are the critical correlators $\phi_{q}^{c}(t)$ for the wave vector $q=4.2$ calculated with the MSA-structure factor for the critical attraction well width $\delta^{*}=0.0465$. The states are located on the transition line as shown in the inset and refer to critical Debye-Waller factors $f_{q}^{c}=0.973,0.910,0.772,0.593,0.460$ (from top to bottom as indicated by horizontal straight lines). For states 1 and $5, \lambda=0.80$ corresponding to a critical exponent $a=0.279$; state 2 (4) refers to $\lambda=0.895(0.908)$ corresponding to $a=0.210(0.202)$. The dash-dotted lines show the asymptotes $f_{q}^{c}+A_{q} t^{-a}$. State 3 is at the $A_{4}$ singularity, given by Eq. (16b).

if one considers states closer to the $A_{4}$ point, the onset of the critical power law gets shifted to larger times. This is demonstrated for the two states 2 and 4 , which deal with $\lambda$ $\approx 0.90$. For the state 4 , the $t^{-a}$ law with $a \approx 0.20$ is valid only for $t>10^{6}$. This trend continues if one moves even closer to the $A_{4}$ point, whereby $\lambda$ increases even further; compare Fig. 6. At the $A_{4}$ singularity, the correlator decays from 1.00 to $f_{q}^{c} \approx 0.77$. This decay is stretched so enormously that even for $t=10^{12}$ it only reaches the value $\phi_{q}(t) \approx 0.80$, as is shown by curve 3 in Fig. 10. One can describe the critical correlator with Eqs. (25) and (26c) for the window $10^{15}<t<10^{25}$, using $h_{q}$ and $h_{q}^{(1)}$ as fit parameters. But this fit does not describe the correlator for $t<10^{10}$. Thus one concludes that the critical correlator of the $A_{4}$ for the SWS cannot be described by the asymptotic Eq. (26c) within accessible dynamical windows. Nor can the critical power-law decay of the $A_{2}$ singularity be measured if $\lambda$ exceeds a certain value, say 0.9 . Thus, there is a part of the transition lines near the $A_{4}$ point, characterized by $\lambda \geqslant 0.9$, where the correlators exhibit structural relaxation patterns towards the plateau values $f_{q}^{c}$ that are stretched up to $t=10^{6}$ or larger. The known asymptotic formulas cannot be used to describe the MCT solutions within this regime.

The liquid dynamics on the small- $\delta$ side of the $A_{4}$ point is particularly subtle, since there is an $A_{3}$ singularity in addition to the line-crossing point. Figure 11 exhibits as an example such a situation for $\delta=0.03$. Parameters on a straight line, $\theta=0.1875$, which is slightly above the $A_{3}$ point, are considered. The transition to a repulsion-dominated glass state then occurs at $\varphi_{c}=0.5360$. At the transition point, the critical Debye-Waller factor is $f_{q}^{(1) c} \approx 0.50$, and the exponent parameter is given by $\lambda=0.847$, implying a critical exponent $a$

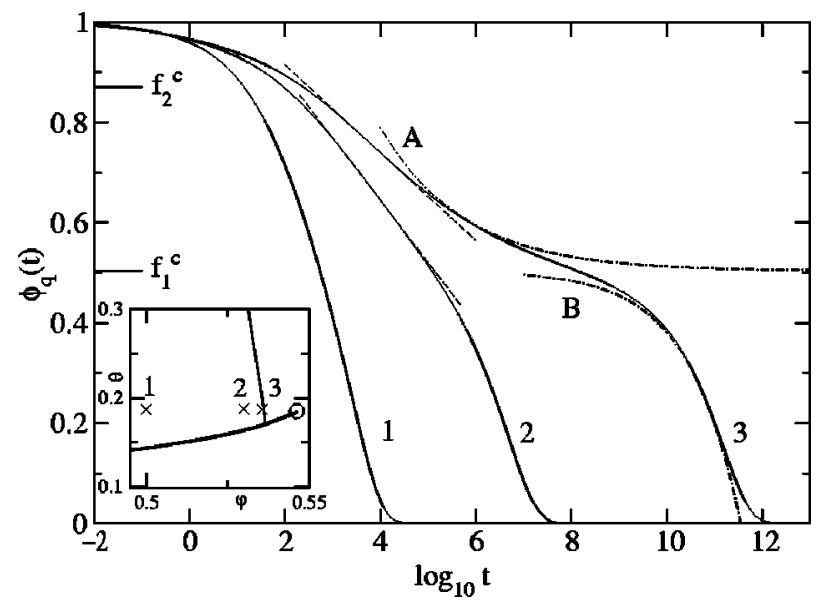

FIG. 11. Correlators $\Phi_{q}(t)$ for $q=4.2$ calculated for the MSA structure factor of a SWS with attraction well width $\delta=0.03$ and the reduced temperature $\theta=0.1875$ for the three packing fractions $\varphi_{1}=0.5000, \varphi_{2}=0.5300, \varphi_{3}=0.5357$ (full lines). The inset shows the relevant section from the phase diagram of Fig. 5(b). The dashed-dotted lines with labels $A$ and $B$ exhibit the critical law $f_{q}^{(1) c}+A_{q} / t^{0.250}$ and the von Schweidler law $f_{q}^{(1) c}-B_{q} t^{0.396}$, respectively. The straight dashed lines exhibit logarithmic decay laws, Eq. (27), see text.

$=0.250$. Curve 3 was calculated for such a small distance from the transition point, $-\varepsilon=\left(\varphi_{c}-\varphi\right) / \varphi_{c}=7.9 \times 10^{-4}$, that $\phi_{q}(t)$ decays to zero just within the dynamical window displayed in the figure. The dash-dotted line with label $A$ presents the leading-order critical law for the $A_{2}$ singularity, Eqs. (25) and (26a), with the time scale $t_{0}$ fitted to the decay at long times for $\varphi=\varphi_{c}$. One observes the same phenomenon as explained above in connection with Fig. 10: since $\lambda$ is rather large, the asymptotic law describes the data only for rather large times, $t>10^{5.5}$. After falling below the plateau value $f_{q}^{(1) c}$, the correlator decays towards zero. This is the $\alpha$ process, and it starts with the von Schweidler asymptote $f_{q}^{(1) c}-h_{q}(t / \tau)^{b}$, which is shown by the dash-dotted line with label $B$. The exponent $b=0.396$ obeys a similar relation as the critical exponent, $\Gamma(1+b)^{2} / \Gamma(1+2 b)=\lambda$. Thus the structural relaxation connected with the liquid-glass transition follows the known scenario, except that the familiar $A_{2}$ patterns can be observed only for times far out of the transient regime, $t>10^{5.5}$.

Figure 11 exhibits a large dynamical window, $10^{2}<t$ $<10^{5.5}$, where the structural relaxation does not follow the asymptotic laws for a fold bifurcation. Instead, the dashed straight line demonstrates that the correlator labeled 3 follows a logarithmic decay law,

$$
\phi_{q}(t)=f_{q}^{(2) c}-C_{q} \ln t
$$

for the major part of the mentioned window, $10^{2} \leqslant t \leqslant 10^{4.5}$. Here, $f_{q}^{(2) c} \approx 0.87$ is the Debye-Waller factor at the $A_{3}$ singularity. There is a line through every $A_{3}$ singularity, which is transversal to the transition line ending at the $A_{3}$, such that Eq. (27) is a leading-order solution for the MCT equations of motion on a certain intermediate time window. The length of the window expands and the prefactor $C_{q}$ in front of the $\ln t$ 


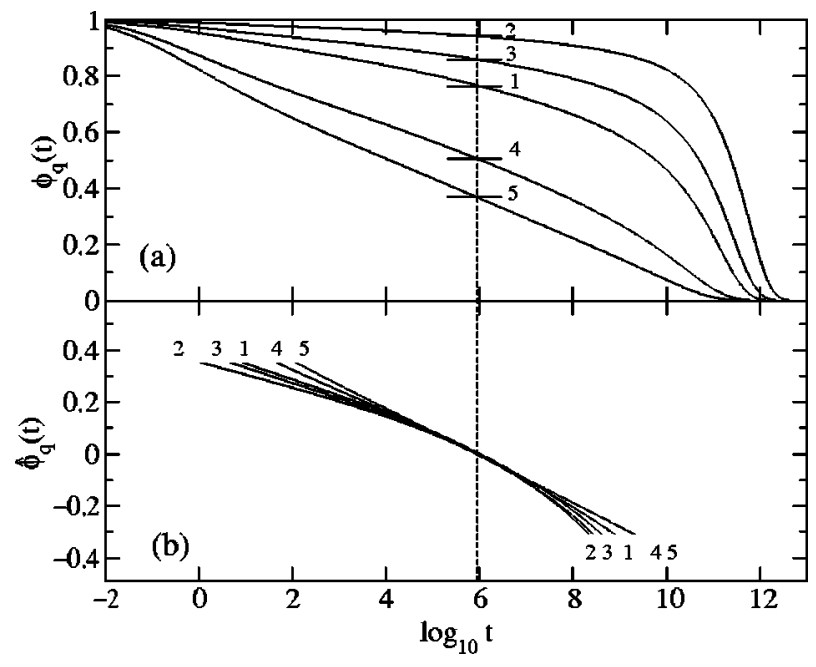

FIG. 12. Correlators for a liquid state close to the $A_{4}$ singularity calculated with the MSA for the structure factor. The curves in the upper panel (a) show the $\phi_{q}(t)$ where the labels 1 to 5 indicate the wave numbers $q=4.2,7.0,8.2,20.2,24.2$. The corresponding critical Debye-Waller factors $f_{q}^{c}$ are 0.764, 0.943, 0.860, 0.507, 0.369, respectively. The curves in the lower panel (b) exhibit the rescaled correlators $\hat{\phi}_{q}(t)=\left[\phi_{q}(t)-f_{q}^{c}\right] / h_{q}$. Here, the critical amplitudes $h_{q}=\left(1-f_{q}^{c}\right)^{2} e_{q}$ have the values $0.4665,0.1343,0.2881,0.7291$, 0.7835 . The dashed vertical line marks the time $t_{-}=8.9 \times 10^{5}$.

decreases, if one moves closer towards the $A_{3}$ point [53]. These results explain the appearance of the $\ln t$ part and the change of its prefactor if one compares curve 2 with curve 3 in Fig. 11. One concludes that it is the bifurcation dynamics of the $A_{3}$ singularity which prevents the evolution of the $t^{-a}$ law for the fold bifurcation. Similarly, the $\alpha$ process for curve 2 does not start with von Schweidler's law. Therefore, contrary to what one observes for the dynamics of the HSS for comparable large times [41], the $\alpha$ processes for curves 2 and 3 do not obey the superposition principle. Close to the $A_{3}$ point, the dynamics outside the transient and preceding the onset of the $\ln t$ decay law follows the critical law for the $A_{3}$, as given by Eqs. (25) and (26b). But for the shown curves, the situation is similar as explained comprehensively for the critical decay for the HSS [41]. The plateau $f_{q}^{(2) c}$ is so high that there is only a small variation remaining for the $1 /(\ln t)^{2}$ law to manifest itself. The correction terms for the cited leading-order and next-to-leading-order contributions are so large that one has to consider states much closer to the $A_{3}$ to see the result of Eq. (26b).

For state 1 , the $A_{2}, A_{3}$, and $A_{4}$ singularities are so far away that none of the cited asymptotic laws is clearly developed. On the other hand, they are close enough to cause a considerable relaxation stretching. The correlator $\phi_{q}(t)$ needs a dynamical window of three orders of magnitude to complete the $80 \%$ of its decay from 0.9 to 0.1 , as is shown in Fig. 11.

In Fig. 12(a) a set of correlators $\phi_{q}(t)$ for five representative wave numbers is shown. The state refers to the liquid close to the $A_{4}$ point. For $q=24.2$ the window for the logarithmic decay extends from $t=10^{3}$ to $t=10^{10}$. For the other wave numbers the corresponding windows are smaller, i.e., the window of validity for the leading-order asymptotic laws depends on the chosen correlator. If the correction term $h_{q}^{(1)} G^{(1)}(t)$ in Eq. (25) could be neglected, i.e., if the factorization theorem $\phi_{q}(t)-f_{q}^{c}=h_{q} G(t)$ would hold, the rescaled correlators $\hat{\phi}_{q}(t)=\left[\phi_{q}(t)-f_{q}^{c}\right] / h_{q}$ should collapse on the common function $G(t)$. In particular, all correlators should cross their plateau value $f_{q}^{c}$ at the same time $t_{-}$, given by $G\left(t_{-}\right)=0$. The latter property is fulfilled within a small error margin for $t_{-}=8.9 \times 10^{5}$. Figure 12 (b) demonstrates the validity of the factorization property for a two-decade window. The size of this window is considerably smaller than the one found for the HSS for a state with a similar overall relaxation time [41]. Thus, the next-to-leading-order correction in Eq. (25) is much larger near the $A_{4}$ than the one known from the bifurcation dynamics of the simple HSS.

\section{CONCLUSIONS}

In this paper ideal liquid-glass transitions and the evolution of glassy dynamics were analyzed within the basic version of the mode-coupling theory (MCT) for a simple colloid model, where the particles interact via a square-well potential. The discussion was restricted to the high-density regime. Hence the excluded-volume effects play a crucial role for the structure, and the cage effect is an essential feature of the dynamics. The presence of short-ranged attractions leads to a variety of new features compared to the ones known from the hard-sphere system (HSS). We find a subtle phase diagram for the glass-transition lines in the plane spanned by the two control parameters, packing fraction $\varphi$, and reduced temperature $\theta$ (Fig. 5). The diagram is organized around an $A_{4}$-glass-transition singularity. This occurs for a critical value $\delta^{*} \approx 0.04$ of the ratio $\delta$ of the attraction-well width and the hard-core diameter, a packing fraction $\varphi^{*}$ exceeding the transition density $\varphi_{c}^{\mathrm{HSS}}$ of the HSS, and a certain critical temperature, cf. Eqs. (16).

For $\delta>\delta^{*}$ and sufficiently low temperature, there is a part of the liquid-glass transition line where the critical temperature $\theta_{c}$ increases with the critical density $\varphi_{c}$. As expected for conventional liquids with, e.g., Lennard-Jones interactions, the glass transition can occur either upon cooling or upon compression. This part of the transition line extends up to densities where $\varphi$ exceeds $\varphi_{c}^{\text {HSS }}$, since the bonding effects due to the attraction stabilize the liquid phase. For large temperatures, the effects of the attraction get suppressed. Therefore, there exists a high-temperature piece of the transition line, where $\theta_{c}$ decreases with increasing $\varphi_{c}$. There appears a regime of high density, where the liquid can transform to a glass either by cooling or by heating. The possibility of such a reentry phenomenon is characteristic for systems with a hard-core repulsion. In a conventional system the effect cannot occur since a soft-core repulsion implies a decrease of the effective repulsion-core diameter with heating, and this decrease overcompensates the effect of the decrease of the effective attraction strength.

For $\delta<\delta^{*}$ the two mentioned transition-line parts no longer join smoothly. Rather, the low-temperature line terminates the high-temperature one at some crossing point, such 
that they appear as two separate transition lines. At very high temperature the mechanism of glass formation is similar to the one of the HSS, and in general, the temperature dependence of the high-temperature transition line is weak. Glass transitions across this line are caused by an arrest of density fluctuations on the length scale of the interparticle distance. A tagged particle is localized due to repulsion by its cageforming neighbors. In contrast, the low-temperature line describes glass formation due to the arrest of density fluctuations on a length scale of the order of the attraction-shell width. Here, tagged particles are localized due to a formation of short bonds with their cage-forming neighbors. The density dependence of these transition points is weak, and the transition line extends into the regime of gel formation at low densities. On the high-density side, it extends into the glass regime, until it ends at an $A_{3}$-glass-transition singularity, as indicated by the open circles in Fig. 5(b).

Between the mentioned line-crossing point and the end point of the second transition line, there is a line of glassglass transitions. The averaged equilibrium structure, as characterized by the structure factor $S_{q}$, is the same on either side of this line. But the two different localization mechanisms imply qualitatively different frozen structures, reflected by differences in the Debye-Waller factor $f_{q}$. The one on the high-temperature side, shown by the uppermost solid line in Fig. 8, is similar to the Debye-Waller factor of the HSS at the same density. It exhibits a pronounced peak near the position $q_{0}$ of the structure-factor peak, and the zero-wave-number limit $f_{0}^{c}$ is about 0.7. On the lowtemperature side $f_{q}^{c}$ is much larger, as is shown by the lowest of the dashed lines in Fig. 8. In particular, $f_{0}^{c}$ is considerably increased. The $f_{q}^{c}$-versus- $q$ curve for the attractiondominated glass is bell-shaped like a Mößbauer-Lamb factor. The increase of $f_{0}^{c}$ towards the upper limit unity is connected with a large enhancement of the longitudinal modulus. Crossing the glass-glass transition line, the longitudinal modulus as well as the shear modulus experiences a large discontinuity, as shown in Fig. 9. The large differences in the macroscopic elastic properties are the most obvious manifestations of the two localization mechanisms in the highdensity system predicted by our theory.

Two general MCT predictions for the relaxation near a critical temperature or critical density have been confirmed by many experiments and molecular-dynamics simulations [54]. First, the structural relaxation exhibits a two-step scenario. Outside the transient, there occurs a relaxation towards the plateau $f_{q}^{c}$. For this step, $d^{2} \phi_{q}(t) / d(\ln t)^{2}$ is positive. Then there is the $\alpha$ process dealing with the relaxation from the plateau to zero. Its initial part exhibits a negative second derivative of the $\phi_{q}(t)$-versus-ln $t$ curve. Second, there holds the superposition principle for the $\alpha$ process. On a time window that expands with increasing relaxation time, the $\phi_{q}(t)$-versus-ln $t$ curves can be collapsed on a common master curve by shifts along the abscissa. These two simple results, which are fingerprints of the $A_{2}$ bifurcation, are not valid for the relaxation at states close to an $A_{4}$ singularity. The curves in Fig. 11 cannot be rescaled onto an $\alpha$-relaxation master curve. The results in Fig. 12 do not ex- hibit changes of the second derivatives for $\phi_{q}(t)$ near the plateau $f_{q}^{c}$. It was shown that the higher-order glasstransition singularities $A_{3}$ and $A_{4}$ cause strong perturbations of the asymptotic laws usually considered, valid close to the $A_{2}$ bifurcation. In the present case they can only be observed in windows, which might be outside the regimes accessible by experiments. In addition, the known asymptotic laws for the relaxation near $A_{3}$ or $A_{4}$ glass-transition singularities also show up only in windows, which are irrelevant for experimental studies. These predictions of our theory do not seem to be a peculiarity of the square-well system. Similar results already hold for simple one-component schematic models [52].

An exception to the findings summarized in the preceding paragraph is the logarithmic-decay law, Eq. (27). This characteristic feature of the dynamics near higher-order glasstransition singularities could be identified easily in our results, as shown in Fig. 12. Indeed, it is shown in Fig. 11 that this $\ln t$ decay is a precursor phenomenon, hindering the evolution of the $A_{2}$ asymptotics. In particular, there can be a crossover from the $\ln t$ decay to the von Schweidler decay around the point where the $\phi_{q}(t)$-versus-ln $t$ curve crosses the plateau $f_{q}^{c}$, as is shown by curve 3 in Fig. 11. A similar scenario was recently observed for relaxation in a micellar system [55].

The found extreme stretching phenomena have important implications for the experimental tests of MCT. In an experiment, it is not easily possible to measure self-averaged correlation functions for states like the ones discussed in Fig. 12. Thus, experimental results are likely to refer to historydependent nonequilibrium states, and aging effects are likely to be more pronounced than they are for the normal liquidglass transition. Even if proper averaging could be achieved, one cannot determine the Debye-Waller factor $f_{q}=\phi_{q}(t$ $\rightarrow \infty$ ) within accessible time windows, if the states are similar to the ones shown with labels 3 and 4 in Fig. 10. Similar conclusions apply for the measurements of the moduli near the glass-glass-transition line.

The presented theory is based on some assumptions which we would like to discuss in more detail. First, one should expect that the equilibrium state of the system in the density regime considered is a crystal rather than the assumed amorphous phase. In experiments for colloids, crystallization is bypassed by chosing a polydispersity $p$ for the particle diameters. Since nucleation rates decrease dramatically with increasing $p$, a choice of $p$ of some percent is sufficient to establish a metastable amorphous state for practically arbitrarily long times. A small $p$ causes only small changes of the calculated structure factors, and thus only small changes in the coupling coefficients entering the MCT equations. Hence a small $p$ will only imply small changes of the presented results. Indeed, it was shown for the HSS that a change of $p$ did not yield detectable changes of the measured $\phi_{q}(t)[56,57]$. But, it is unclear how strongly, e.g., the calculated value $\delta^{*}$ for the attraction-well width at the $A_{4}$ singularity will change, if a realistic value for $p$ is considered.

The structure factor $S_{q}$ of the stable or metastable equilibrium is used as input information for our work. Thus, the second source of reservations is due to the errors hidden in 
the used $S_{q}$. A well-known problem is that of the so-called thermodynamic inconsistency. Thermodynamic quantities calculated along different routes using an approximate $S_{q}$ as input often are not consistent with each other. Sophisticated closures involving adjustable parameters could be used to overcome this problem [4]. Alas, since thermodynamics deals with the $q \rightarrow 0$ limit, for which the phase volume in the mode-coupling integrals becomes small, one would gain no further insight carrying out our calculations of Secs. III and IV using an improved closure for $S_{q}$. For the HSS, one finds only minor changes in the numerical values for the transition points [52,58], and the same is anticipated for the SWS. A further difficulty arises regarding the small- $r$ limit of $c(r)$ and $g(r)$. Due to the approximations introduced for $Q(r)$, one cannot guarantee that the excluded-volume effect, $g(r$ $<d)=0$, is exactly reproduced. In fact, we find that $c(r)$ and thus $g(r)$ develop a pole $A / r$, cf. Eq. (12). Since $g(r)$ is a distribution and since $A / r$ is integrable in three dimensions, an $A / r$ term is to be viewed as small, provided $A$ is small. In the original work on the sticky hard spheres [27], $A$ $=\mathcal{O}\left(K^{2} \varphi\right)$. In our solution, $A=\mathcal{O}(K \delta \varphi)$. The limits $r \rightarrow 0$ and $\delta \rightarrow 0$ do not commute, and our analytical solution decreases the error from a $\delta^{0}$ to a $\delta^{1}$ effect. Since our results based on the Percus-Yevick closure and on the meanspherical approximation are in semiquantitative agreement, we anticipate that better theories for $S_{q}$ will not change the qualitative results of our theory.

Third, the range of applicability of the MCT is not understood. One can use the successful tests of the theory by the experiments performed on hard-sphere colloids, which were cited in Sec. I, as an a posteriori justification of the MCT. But it is not clear whether or not this theory can handle the effects caused by the formation of strong short bonds. On the other hand, the phenomenon of liquid stabilization due to bond formation and the resulting reentry effect, as well as the drastic changes of the elastic properties at the glass-glass transition, seem very plausible. The fact that the MCT brings out these subtleties might be considered as an argument in favor of this approach. In summary, it is the intention of this paper to point out the possibility of new features of glassy dynamics and to suggest a search for these features by experiments on colloids.

\section{ACKNOWLEDGMENTS}

The work of F.S. and P.T. was supported by PRIN97MURST and PRA-HOP-INFM, the work of M.F. by the Deutsche Forschungsgemeinschaft Grant No. Fu 309/3, and the work of Th.V. by Verbundprojekt No. BMBF 03G05TUM.

\section{APPENDIX: THE MSA FACTOR FUNCTION}

\section{General formulas}

Starting from Eqs. (4) and (5), Eqs. (9) for the factor function and expressions (10) and (11) for the parameters $a, b, c$ shall be derived. The region $0<r<1+\delta$, for which $Q(r)$ is nonzero, can be split into three parts,

$$
Q(r)= \begin{cases}q_{\mathrm{I}}(r), & 0<r<\delta \\ q_{\mathrm{II}}(r), & \delta<r<1 \\ q_{\mathrm{III}}\left(r^{\prime}\right), & 0<r^{\prime}=r-1<\delta .\end{cases}
$$

$Q(r)$ is continuous at the boundaries of the intervals, in particular $Q(1+\delta)=0$. From Eq. (5), together with $g(r)=1$ $+h(r)$ and $G\left(r^{\prime}\right)=\left(1+r^{\prime}\right) g\left(1+r^{\prime}\right)$, the derivatives for the three parts of the factor function are obtained

$$
\begin{gathered}
q_{\mathrm{I}}^{\prime}(r)=a r+b-12 \varphi \int_{r}^{\delta} d s G(s-r) q_{\mathrm{III}}(s), \\
q_{\mathrm{II}}^{\prime}(r)=a r+b, \\
q_{\mathrm{III}}^{\prime}\left(r^{\prime}\right)=a r+b-G\left(r^{\prime}\right)+12 \varphi \int_{0}^{r^{\prime}} d s G\left(r^{\prime}-s\right) q_{\mathrm{I}}(s) .
\end{gathered}
$$

Here $g(r)=0$ for $0<r<1$ was used, and the definition for $a$ and $b$ is given in Eq. (6b). The integrated form of Eq. (4) is used to introduce the MSA closure as in Eq. (8),

$$
\begin{aligned}
q_{\mathrm{III}}\left(r^{\prime}\right)= & 12 \varphi \int_{r^{\prime}}^{\delta} d s q_{\mathrm{III}}(s) q_{\mathrm{I}}\left(s-r^{\prime}\right) \\
& +K\left[1-\frac{r^{\prime}}{\delta}+\frac{\delta}{2}\left(1-\frac{r^{\prime 2}}{\delta^{2}}\right)\right],
\end{aligned}
$$

where $K=u_{0} \delta / k_{B} T$. In the following, Eqs. (A2) and (A3) are solved together with the reformulated expressions for $a$ and $b$

$$
\begin{aligned}
a= & 1-12 \varphi\left[\int_{0}^{\delta} d s q_{\mathrm{I}}(s)+\int_{\delta}^{1} d s q_{\mathrm{II}}(s)+\int_{0}^{\delta} d s q_{\mathrm{III}}(s)\right], \\
b= & 12 \varphi\left[\int_{0}^{\delta} d s s q_{\mathrm{I}}(s)+\int_{\delta}^{1} d s s q_{\mathrm{II}}(s)+\int_{0}^{\delta} d s s q_{\mathrm{III}}(s)\right. \\
& \left.+\int_{0}^{\delta} d s q_{\mathrm{III}}(s)\right] .
\end{aligned}
$$

Equation (A2b) gives Eq. (9a),

$$
q_{\mathrm{II}}(r)=a r^{2} / 2+b r+c,
$$

where the continuity $q_{\mathrm{II}}(r=1)=q_{\mathrm{III}}\left(r^{\prime}=0\right)$ yields $c$. For intervals I and III an expansion in $\delta$ for fixed $K$ will be performed.

\section{Leading order}

In Eq. (A3), $r^{\prime} / \delta$ is of order $\delta^{0}$ and the integral is of higher order $\delta^{1}$. Therefore, in leading order,

$$
q_{\mathrm{III}}\left(r^{\prime}\right)=K\left(1-r^{\prime} / \delta\right) .
$$

The boundary condition mentioned above fixes $c=K-a / 2$ $-b$ in leading order. Substituting Eq. (A6) into Eq. (A2c) 
and keeping only terms in lowest order in $\delta$ results in $G\left(r^{\prime}\right)=K / \delta$. The integral in Eq. (A2a) is again of higher order and the continuity at the boundary $r=\delta$ gives the parabola also for region $\mathrm{I}$,

$$
q_{\mathrm{I}}(r)=a r^{2} / 2+b r+c .
$$

Inserting the factor function into Eqs. (A4) and keeping only lowest-order terms, linear equations for the parameters are obtained

$$
\begin{gathered}
a=1-12 \varphi(a / 6+b / 2+c), \\
b=12 \varphi(a / 8+b / 3+c / 2),
\end{gathered}
$$

which leads to Eqs. (10).

\section{Next-to-leading order}

Substituting the leading-order results into Eqs. (A2) and (A3) produces the next-to-leading order. Subtracting Eq. (A2b) from Eq. (A2a), the result for the interval I is given as the derivative

$$
\begin{aligned}
q_{\mathrm{I}}^{\prime}(r)-q_{\mathrm{II}}^{\prime}(r) & =-12 \varphi \int_{r}^{\delta} d s K / \delta K(1-s / \delta) \\
& =-6 \varphi K^{2}(1-r / \delta)^{2}
\end{aligned}
$$

which is integrated to give the last term in Eq. (9b). In Eq. (A3) the entire last line has to be taken into account for the next-to-leading order. The integral reads

$$
12 \varphi \int_{r^{\prime}}^{\delta} d s q_{\mathrm{III}}(s) q_{\mathrm{I}}\left(s-r^{\prime}\right)=6 K \delta \varphi c_{0}\left(1-r^{\prime} / \delta\right)^{2}+\mathcal{O}\left(\delta^{2}\right)
$$

Combinig Eqs. (A3) and (A10) yields the expression for the next-to-leading order term for the factor function in the outer shell, Eq. (9c). The continuity at $r=1$ introduces a modification of $c$ from its leading-order value $c_{0}$,

$$
c=K-a / 2-b+\delta K / 2+6 \delta K \varphi c_{0},
$$

where $a$ and $b$ are given by inserting the factor functions into Eqs. (A4),

$$
a=1-12 \varphi(a / 6+b / 2+c+\delta K / 2),
$$

$$
b=12 \varphi(a / 8+b / 3+c / 2+\delta K / 2) .
$$

This yields Eqs. (9), (10), and (11).
[1] W. B. Russel, D. A. Saville, and W. R. Schowalter, Colloidal Dispersions (Cambridge University Press, New York, 1989).

[2] P. N. Pusey, in Liquids, Freezing and Glass Transition, edited by J.-P. Hansen, D. Levesque, and J. Zinn-Justin (NorthHolland, Amsterdam, 1991), p. 763.

[3] W. C. K. Poon, Curr. Opin. Colloid Interface Sci. 3, 593 (1998).

[4] J. P. Hansen and I. R. McDonald, Theory of Simple Liquids (Academic Press, London, 1986).

[5] U. Balucani and M. Zoppi, Dynamics of the Liquid State (Clarendon Press, Oxford, 1994).

[6] W. van Megen and P. N. Pusey, Phys. Rev. A 43, 5429 (1991).

[7] S. F. Edwards and P. W. Anderson, J. Phys. F: Met. Phys. 5, 965 (1975)

[8] W. van Megen, S. M. Underwood, and P. N. Pusey, Phys. Rev. Lett. 67, 1586 (1991).

[9] W. van Megen and S. M. Underwood, Phys. Rev. Lett. 70, 2766 (1993).

[10] W. van Megen and S. M. Underwood, Phys. Rev. E 47, 248 (1993)

[11] W. van Megen and S. Underwood, Phys. Rev. Lett. 72, 1773 (1994).

[12] W. van Megen and S. M. Underwood, Phys. Rev. E 49, 4206 (1994).

[13] W. van Megen, T. C. Mortensen, S. R. Williams, and J. Müller, Phys. Rev. E 58, 6073 (1998).

[14] U. Bengtzelius, W. Götze, and A. Sjölander, J. Phys. C 17, 5915 (1984).

[15] E. Leutheusser, Phys. Rev. A 29, 2765 (1984).
[16] W. Götze and L. Sjögren, Rep. Prog. Phys. 55, 241 (1992).

[17] W. van Megen, Transp. Theory Stat. Phys. 24, 1017 (1995).

[18] T. G. Mason and D. A. Weitz, Phys. Rev. Lett. 75, 2770 (1995).

[19] E. Bartsch, M. Antonietti, W. Schupp, and H. Sillescu, J. Chem. Phys. 97, 3950 (1992).

[20] E. Bartsch, V. Frenz, S. Müller, and H. Sillescu, Physica A 201, 363 (1993).

[21] E. Bartsch, J. Non-Cryst. Solids 192-193, 384 (1995).

[22] E. Bartsch, V. Frenz, and J. Baschnagel, J. Chem. Phys. 106, 3743 (1997).

[23] Ch. Beck, W. Härtl, and R. Hempelmann, J. Chem. Phys. 111, 8209 (1999).

[24] L. Fabbian, W. Götze, F. Sciortino, P. Tartaglia, and F. Thiery, Phys. Rev. E 59, R1347 (1999); 60, 2430 (1999).

[25] J. Bergenholtz and M. Fuchs, Phys. Rev. E 59, 5706 (1999).

[26] G. Foffi, E. Zaccarelli, F. Sciortino, P. Tartaglia, and K. A. Dawson, J. Stat. Phys. 100, 363 (2000).

[27] R. J. Baxter, J. Chem. Phys. 49, 2770 (1968); in Physical Chemistry. An Advanced Treatise, edited by D. Henderson (Academic Press, New York, 1971), Vol. VIIIA, p. 267.

[28] W. C. K. Poon, J. S. Selfe, M. B. Robertson, S. M. Ilett, A. D. Pirie, and P. N. Pusey, J. Phys. II 3, 1075 (1992).

[29] H. N. W. Lekkerkerker, W. C. K. Poon, P. N. Pusey, A. Stroobants, and P. B. Warren, Europhys. Lett. 20, 559 (1992).

[30] A. Meller, T. Gisler, D. A. Weitz, and J. Stavans, Langmuir 15, 1918 (1999).

[31] M. C. Grant and W. B. Russel, Phys. Rev. E 47, 2606 (1993).

[32] H. Verduin and J. K. G. Dhont, J. Colloid Interface Sci. 172, 425 (1995) 
[33] C. J. Rueb and C. F. Zukoski, J. Rheol. 41, 197 (1997).

[34] C. J. Rueb and C. F. Zukoski, J. Rheol. 42, 1451 (1998).

[35] L. Lobry, N. Micali, F. Mallamace, C. Liao, and S.-H. Chen, Phys. Rev. E 60, 7076 (1999).

[36] W. G. T. Kranendonk and D. Frenkel, Mol. Phys. 64, 403 (1988).

[37] A. Lang, G. Kahl, C. N. Likos, H. Löwen, and M. Watzlawek, J. Phys.: Condens. Matter 11, 10143 (1999).

[38] R. J. Baxter, Aust. J. Phys. 21, 563 (1968).

[39] I. Nezbeda, Czech. J. Phys., Sect. B 24, 703 (1974).

[40] I. Nezbeda, Czech. J. Phys., Sect. B 27, 247 (1977).

[41] T. Franosch, M. Fuchs, W. Götze, M. R. Mayr, and A. P. Singh, Phys. Rev. E 55, 7153 (1997).

[42] W. Götze, in Liquids, Freezing and Glass Transition, edited by J.-P. Hansen, D. Levesque, and J. Zinn-Justin (North-Holland, Amsterdam, 1991), p. 287.

[43] W. Götze and L. Sjögren, J. Math. Anal. Appl. 195, 230 (1995).

[44] V. I. Arnol'd, Catastrophe Theory (Springer, Berlin, 1992).

[45] M. Fuchs, W. Götze, and M. R. Mayr, Phys. Rev. E 58, 3384 (1998).

[46] J. Bergenholtz, M. Fuchs, and Th. Voigtmann, J. Phys. Con- dens. Matter 12, 6575 (2000).

[47] J. Bergenholtz and M. Fuchs, J. Phys.: Condens. Matter 11, 10171 (1999).

[48] T. Franosch, W. Götze, M. R. Mayr, and A. P. Singh, J. NonCryst. Solids 235-237, 71 (1998).

[49] M. Fuchs and Th. Voigtmann, Philos. Mag. B 79, 1799 (1999).

[50] M. Fuchs and M. R. Mayr, Phys. Rev. E 60, 5742 (1999).

[51] W. Götze and L. Sjögren, J. Phys.: Condens. Matter 1, 4203 (1989).

[52] M. Sperl, Diploma thesis, Technische Universität München, 2000 (unpublished).

[53] W. Götze and R. Haussmann, Z. Phys. B: Condens. Matter 72, 403 (1988).

[54] W. Götze, J. Phys.: Condens. Matter 11, A1 (1999).

[55] F. Mallamace, P. Gambadauro, N. Micali, P. Tartaglia, C. Liao, and S.-H. Chen, Phys. Rev. Lett. 84, 5431 (2000).

[56] S. I. Henderson, T. C. Mortensen, G. M. Underwood, and W. van Megen, Physica A 233, 102 (1996).

[57] S. I. Henderson and W. van Megen, Phys. Rev. Lett. 80, 877 (1998).

[58] J.-L. Barrat, W. Götze, and A. Latz, J. Phys.: Condens. Matter 1, 7163 (1989). 